\title{
Optimum sampling in spatial-temporally correlated wireless sensor networks
}

\author{
Ning Sun and Jingxian $\mathrm{Wu}^{*}$
}

\begin{abstract}
The optimum sampling in the one- and two-dimensional (1-D and 2-D) wireless sensor networks (WSNs) with spatial-temporally correlated data is studied in this article. The impacts of the node density in the space domain, the sampling rate in the time domain, and the space-time data correlation on the network performance are investigated asymptotically by considering a large network with infinite area but finite node density and finite temporal sampling rate, under the constraint of fixed power per unit area. The impact of space-time sampling on network performances is investigated in two cases. The first case studies the estimations of the space-time samples collected by the sensors, and the samples are discrete in both the space and time domains. The second case estimates an arbitrary data point on the space-time hyperplane by interpolating the discrete samples collected by the sensors. Optimum space-time sampling is obtained by minimizing the mean square error distortion at the network fusion center. The interactions among the various network parameters, such as spatial node density, temporal sampling rate, measurement noise, channel fading, and their impacts on the system performance are quantitatively identified with analytical and numerical studies.
\end{abstract}

Keywords: Wireless sensor network, Space-time correlation, Sensor density, Space-time sampling

\section{Introduction}

Data collected by a wireless sensor network (WSN) often contain redundancy due to the spatial and temporal correlation inherent in the monitored object(s). The spatialtemporal data correlations can be found in a wide range of practical applications, such as environment monitoring with temperature and humidity correlated in the space and time domains, soil and water quality monitoring with the chemical compositions correlated in the space and time domains, and structure health monitoring with spatial-temporally correlated vibration information of the civil structure [1], etc. The space-time redundancy/correlation is important to the performance and design of practical WSNs, which attempt to reconstruct a spatial-temporally correlated signal field by collecting the data samples from the sensors. Given a fixed transmission power per unit area, a higher spatial node density or temporal sampling rate means less transmission energy per sample, which usually degrades performance due to a lower signal-to-noise ratio (SNR) at the receiver. On the

*Correspondence: wuj@uark.edu

Department of Electrical Engineering, University of Arkansas, Fayetteville, AR 72701 USA other hand, the system performance might benefit from more data samples per unit area per unit time by exploiting the space-time redundancy. Therefore, it is critical to identify the optimum space-time sampling, i.e., the optimum spatial node density and temporal sampling rate, in a WSN with spatial-temporally correlated data.

There have been considerable works in the literature studying the impacts of spatial node density on the network performance [2-6]. In [2], the optimum node density of a many-to-one linear network is analyzed by using the detection probability of a binary event as the performance metric. In [3], a Wiener process is used to model the spatial correlation of an one-dimensional (1-D) field. It is demonstrated that, due to the spatial data correlation, distortion-free communication can be achieved even if the per node throughput tends to 0 as $N \rightarrow \infty$. The optimum node densities in both 1-D and two-dimensional (2-D) networks are obtained by minimizing the mean square error (MSE) between the recovered information and the original information under a distortiontolerant communication framework [5,6]. Most existing studies focus only on the spatial data correlation, and they do not consider the variation of the data in the time domain. In reality, the physical phenomenon under

\section{Springer}

(c) 2013 Sun and Wu: licensee Springer. This is an Open Access article distributed under the terms of the Creative Commons Attribution License (http://creativecommons.org/licenses/by/2.0), which permits unrestricted use, distribution, and reproduction in any medium, provided the original work is properly cited. 
monitoring changes with respect to time, and the consecutive observations of a sensor node are often correlated temporally [7].

There are limited works on the study of WSNs with spatial-temporally correlated data [8-12]. In [8], an arbitrary point on a continuous measurement field is estimated by performing space-time interpolation over the samples collected by the spatially discrete sensors, and there is a finite optimum node density to minimize the estimation MSE over the measurement field. The model in [8] is extended in [9] by considering realistic transmission schemes, such as a limited transmission range and practical network/routing parameters. In [8,9], the temporal data correlation is only utilized to perform time domain interpolation, and they do not consider the effects of optimum time domain sampling. The effects of both space and time domain sampling are studied in [10] by using the network energy as a performance metric, through the study of a collision free network protocols. All of the aforementioned studies consider an error-free communication channel between the transmitter and the receiver. The impacts of additive white Gaussian noise (AWGN) are considered in [11], which obtains a lower bound on the distortion as a function of the number of sensors and spatial-temporal communication bandwidth. However, the analysis is only applicable to a measurement field with finite degree-of-freedom and is discrete in the time domain. In addition, it does not consider the optimum sampling rate in the time domain. The optimum spacetime sampling of continuous data in an 1-D network with AWGN channel is studied in [12].

In this article, we investigate the optimum space-time sampling for both 1-D and 2-D WSNs with spatialtemporally correlated data. The 1-D network can be used to model practical WSNs designed for highways and tunnels. The 2-D network models WSNs that cover a large area, such as a farmland. There is no limitation on the statistical properties of the field, other than that it forms a continuous random process that is wide sense stationary (WSS) in both the space and time domains. Each sensor node collects samples of the field, and forwards the information to a data fusion center (FC) through an one-hop AWGN or fading channel. Similar one-hop network structures are used in $[2,5,6,12-15]$. The FC attempts to reconstruct the time-varying and spatially continuous data field from the discrete sensor samples by exploiting the data correlation in both the space and time domains with the minimum mean square error (MMSE) receiver. The impacts of the spatial node density, the temporal sampling rate, and the space-time data correlation on the reconstruction MSE are investigated asymptotically in a large network with infinite area, infinite time period, but finite node density and finite temporal sampling rate, under the constraint of fixed transmission power per unit area.
Compared to existing studies in the literature, this article has the following main contributions. First, to the best of our knowledge, this article is the first that explicitly quantifies the interactions between the performance of networks with spatial-temporally correlated data and various system parameters, such as spatial node density, temporal sampling rate, measurement noise, and channel distortions, for both 1-D and 2-D networks. Second, the optimum spatial-temporal sampling for two types of networks, one needs to recover only the discrete space-time samples collected by the sensors through their noisy observations, and one needs to recover an arbitrary data point on the space-time hyperplane, are identified through the asymptotic analysis. Third, the impacts of various practical factors, such as measurement noise, channel fading, and random network topology, on the performance of networks with spatial-temporally correlated data are studied through numerical analysis and simulations.

The remainder of this article is organized as follows. Section 2 introduces the system model and a two-step MMSE estimation method. Sections 3 and 4 studies the impacts of spatial-temporal sampling on 1-D and 2-D networks, respectively, by following the two-step MMSE method. In these two sections, the optimum spatialtemporal samplings in various networks are identified with asymptotic analysis and simulations. Both analytical and numerical results are presented in Sections 3 and 4 to demonstrate the interactions among the various system parameters. Section 5 concludes the article.

\section{Problem formulation}

\subsection{System model}

Consider a WSN with $N_{s}$ sensor nodes uniformly placed over a measurement field. Data collected by the sensors are spatially correlated, and they change with respect to time. We first study a network with a deterministic topology, where the sensors are placed over an equal-distance grid as shown in Figure 1, with the distance between two adjacent nodes being $d$. Such a deterministic topology can be used to model networks that can be carefully planned beforehand and has no limitation on sensor locations. The performance of networks with deterministic topology will be compared to those with randomly distributed nodes. Networks with random topology can be used to model ad hoc networks or networks with mobile nodes. The results obtained for these two types of networks can serve as performance bounds for practical networks, which usually use a combination of these two topologies.

Each sensor node collects data samples with a sampling rate of $\theta=\frac{1}{T_{S}} \mathrm{~Hz}$. In the space domain, define the spatial node density, $\delta$, as the number of nodes in a unit area. The spatial node densities are $\delta=\frac{1}{d}$ and $\delta=\frac{1}{d^{2}}$ for the grid-based 1-D and 2-D networks, respectively. Let 

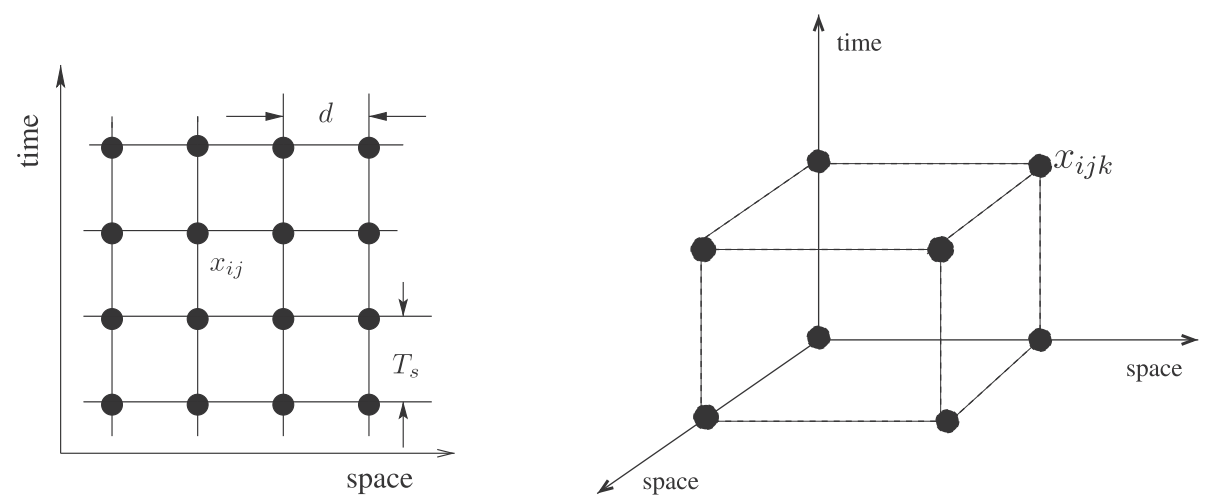

Figure 1 The spatial-temporally correlated sensor networks. (a) The 1-D sensor network with 2-D space-time samples. (b) The 2-D sensor network with 3-D space-time samples.

$\eta=\left[\mathbf{c}^{T}, t\right]^{T}$ represent the coordinate in the space-time hyperplane, where $\mathbf{A}^{T}$ denotes matrix transpose, $\mathbf{c}$ is the coordinate vector in the space domain, and $t$ is the time variable.

Each sensor node will measure a spatial-temporally dependent physical quantity, $x\left(\boldsymbol{\eta}_{\boldsymbol{n}}\right)$, such as the temperature, humidity, or the vibration density of a bridge, etc. It is assumed that the physical quantities to be measured form a random process that is WSS in both the space and time domains. Due to the spatial-temporal redundancy of the measurement field, the spatial-temporal correlation function between any two arbitrary data samples is assumed as

$$
\mathbb{E}\left[x\left(\boldsymbol{\eta}_{1}\right) x\left(\boldsymbol{\eta}_{2}\right)\right]=\rho_{s}^{\left\|\mathbf{c}_{1}-\mathbf{c}_{2}\right\|} \cdot \rho_{t}^{\left|t_{1}-t_{2}\right|}
$$

where $\boldsymbol{\eta}_{n}=\left[\mathbf{c}_{n}^{T}, t_{n}\right]^{T}, \rho_{s} \in[0,1]$ and $\rho_{t} \in[0,1]$ are defined as the spatial correlation coefficient and the temporal correlation coefficient, respectively, and $\mathbb{E}(\cdot)$ represents mathematical expectation. In (1), the $l_{2}$ norm $\left\|\mathbf{c}_{1}-\mathbf{c}_{2}\right\|$ measures the Euclidean distance between the two points with the coordinates $\mathbf{c}_{1}$ and $\mathbf{c}_{2}$ in the space domain.

It is assumed that sensors deliver the measured data to the FC through an orthogonal media access control (MAC) scheme, such as the deterministic frequency division multiple access (FDMA), or the random exponentially-interval MAC (EI-MAC) [16], such that collision-free communication is achieved at the FC. The signal observed by the FC from the $n$th data sample is

$$
y_{n}=\sqrt{\frac{E_{n}}{1+\sigma_{w}^{2}}} \cdot h\left(\boldsymbol{\eta}_{n}\right) \cdot\left[x\left(\boldsymbol{\eta}_{n}\right)+w_{n}\right]+z_{n},
$$

where $E_{n}$ is the average transmission energy per sample, $h\left(\boldsymbol{\eta}_{n}\right)$ represents the quasi-static fading coefficient, $w_{n}$ is the measurement noise with variance $\sigma_{w}^{2}$, and $z_{n}$ is the AWGN with variance $\sigma_{z}{ }^{2}$. It is assumed that the total power per unit area is fixed at $P_{0}$. Given a network with a node density $\delta$ and a sample rate $\theta$, the transmission energy per sample can be calculated as $E_{n}=\frac{P_{0}}{\theta \delta}$. It is assumed here that the sensor-FC distance is much larger than the sensor-sensor distance, such that all the sensors have approximately the same distance to the FC. Therefore, signals from all the sensors experience similar pathloss, such that they can employ the same transmission energy.

\subsection{Optimum MMSE detection}

The FC will obtain an estimate of the spatial-temporally continuous quantity, $x(\boldsymbol{\eta}), \forall \boldsymbol{\eta} \in \Omega_{\eta}$, by using $N=N_{s} N_{t}$ discrete space-time samples received at the FC, where $N_{s}$ is the number of the sensor nodes and $N_{t}$ is the number of time-domain samples collected by each node. Define the space-time data sample vector as $\mathbf{x}_{s t}=\left[\mathbf{x}_{1}^{T}, \ldots, \mathbf{x}_{N_{s}}^{T}\right]^{T} \in$ $\mathcal{R}^{N \times 1}$, where $\mathbf{x}_{i}=\left[x_{i 1}, \ldots, x_{i N_{t}}\right]^{T} \in \mathcal{R}^{N_{t} \times 1}$ is the time domain sample vector collected by the $i$ th sensor node, and $\mathcal{R}$ is the set of real numbers. The corresponding signal observed by the FC can then be represented as $\mathbf{y}=\left[\mathbf{y}_{1}^{T}, \ldots, \mathbf{y}_{N_{s}}^{T}\right]^{T} \in \mathcal{R}^{N \times 1}$, with $\mathbf{y}_{i}=\left[y_{i 1}, \ldots, y_{i N_{t}}\right]^{T} \in$ $\mathcal{R}^{N_{t} \times 1}$.

The MSE for $x(\boldsymbol{\eta})$ is

$$
\sigma_{\eta}^{2}=\mathbb{E}[\hat{x}(\boldsymbol{\eta})-x(\boldsymbol{\eta})]^{2}, \boldsymbol{\eta} \in \Omega_{\eta}
$$

where $\hat{x}(\boldsymbol{\eta})$ is the estimate of $x(\boldsymbol{\eta})$ based on $\mathbf{y}$ at the FC.

The optimum linear receiver that minimizes $\sigma_{\eta}^{2}$ is the MMSE receiver described as follows [17]

$$
\begin{aligned}
\hat{x}(\boldsymbol{\eta})= & \sqrt{\frac{E_{n}}{1+\sigma_{w}^{2}}} \mathbf{r}_{\eta}^{H} \mathbf{H}^{H} \\
& \times\left[\frac{E_{n}}{1+\sigma_{w}^{2}} \mathbf{H R}_{x x} \mathbf{H}^{H}+\frac{E_{n} \sigma_{w}^{2}}{1+\sigma_{w}^{2}} \mathbf{H H}^{H}+\sigma_{z}^{2} \mathbf{I}_{N}\right]^{-1} \mathbf{y},
\end{aligned}
$$


where $\mathbf{r}_{\eta}=\mathbb{E}\left[x(\boldsymbol{\eta}) \mathbf{x}_{s t}\right] \in \mathcal{R}^{N \times 1}, \mathbf{R}_{x x}=\mathbb{E}\left[\mathbf{x}_{s t} \mathbf{x}_{s t}^{H}\right] \in$ $\mathcal{R}^{N \times N}$ with the element defined in (1), and $\mathbf{A}^{H}$ denotes the matrix Hermitian operation. The channel coefficient matrix, $\mathbf{H} \in \mathcal{C}^{N \times N}$, is a diagonal matrix with the diagonal elements being $\mathbf{h}=\left[\mathbf{h}_{1}^{T}, \ldots, \mathbf{h}_{N_{s}}^{T}\right]^{T} \in \mathcal{C}^{N \times 1}$, where $\mathbf{h}_{i}=$ $h_{i} \mathbf{I}_{N_{t}} \in \mathcal{C}^{N_{t} \times 1}$ with $h_{i}$ corresponding to the fading coefficient between the $i$ th node and the FC, $\mathbf{I}_{N_{t}}$ is a size- $N_{t}$ identity matrix, and $\mathcal{C}$ is the set of complex numbers.

With the optimum MMSE receiver given in (4), the MSE $\sigma_{\eta}^{2}$ can be calculated as

$$
\begin{aligned}
\sigma_{\eta}^{2}=\mathbb{E}_{\mathbf{H}}\left\{1-\mathbf{r}_{\eta}^{H}\left[\mathbf{R}_{x x}+\sigma_{w}^{2}\right.\right. \\
\left.\left.+\left(1+\sigma_{w}^{2}\right) \frac{\theta_{0} \delta}{\gamma_{0}}\left(\mathbf{H}^{H} \mathbf{H}\right)^{-1}\right]^{-1} \mathbf{r}_{\eta}\right\}
\end{aligned}
$$

where $\gamma_{0}=\frac{P_{0}}{\sigma_{z}^{2}}$ is the signal-to-noise ratio (SNR) per unit area with AWGN, and the expectation operation is performed with respect to $\mathbf{H}$. The MSE $\sigma_{\eta}^{2}$ given in (5) is a function of the space-time coordinate $\eta$, the SNR $\gamma_{0}$, the measurement noise variance $\sigma_{w}^{2}$, the spatial correlation coefficient $\rho_{s}$, the temporal correlation coefficient $\rho_{t}$, the spatial node density $\delta$, the temporal sampling rate $\theta$, and the fading coefficient $\mathbf{H}$.

Given a fixed transmission power per unit area, the spatial-temporal sampling rate, $\delta$ and $\theta$, play a critical role on the MSE $\sigma_{\eta}^{2}$. A smaller node density and/or temporal sampling rate means more transmission energy per sample, thus a better SNR per sample, which can benefit the system performance. On the other hand, a smaller node density and/or sampling rate means less samples per unit area per unit time, thus a smaller correlation among the data collected by the nodes, and this might degrade the estimation performance.

In order to distinguish the opposite impacts of the spatial-temporal sampling rates, we use an equivalent two-step MMSE method [6].

Lemma 1. The optimum MMSE given in (4) is equivalent to the two-step MMSE described as follows.

1) The FC first obtains an estimate of the $N$ discrete space-time samples, $\mathbf{x}_{s t}$, with a linear MMSE receiver as

$$
\hat{\mathbf{x}}_{s t}=\mathbf{W}_{x}{ }^{H} \mathbf{y}
$$

where $\hat{\mathbf{x}}_{s t} \in \mathcal{R}^{N \times 1}$ is the MMSE estimate of $\mathbf{x}_{s t}$. The MMSE matrix $\mathbf{W}_{x} \in \mathcal{R}^{N \times N}$ is designed to minimize the average MSE per sample:

$$
\sigma_{s t, N}^{2}=\frac{1}{N} \mathbb{E}\left[\left\|\hat{\mathbf{x}}_{s t}-\mathbf{x}_{s t}\right\|^{2}\right]
$$

2) The FC obtains an estimate of the data at an arbitrary location, $\hat{x}(\boldsymbol{\eta}), \forall \boldsymbol{\eta} \in \Omega_{\boldsymbol{\eta}}$, by interpolating $\hat{\mathbf{x}}_{\boldsymbol{s}}$ with the MMSE criterion,

$$
\hat{x}(\boldsymbol{\eta})=\mathbf{w}_{s l}^{H} \hat{\mathbf{x}}_{s t},
$$

where the vector, $\mathbf{w}_{s l} \in \mathcal{R}^{N \times 1}$, is designed to minimize the MSE $\sigma_{\eta}^{2}=\mathbb{E}[\hat{x}(\boldsymbol{\eta})-x(\boldsymbol{\eta})]^{2}$.

Decomposing the optimum MMSE of (4) into the twostep MMSE allows us to study the two opposite effects of spatial-temporal sampling on the MSE separately. In the following two sections, we will investigate, respectively, the impacts of the node density on 1-D and 2-D networks by following the two-step MMSE.

\section{Optimum space-time sampling in one-dimensional networks}

In this section, we study the optimum space-time sampling in an 1-D network, where the $N_{s}$ sensor nodes are evenly distributed over a length- $L$ linear section as shown in Figure 1a. In this WSN, the space-time coordinate of the $j$ th data sample collected by the $i$ th sensor can then be represented as $\left[(i-1) d,(j-1) T_{s}\right]$. The spatial-temporal correlation matrix, $\mathbf{R}_{x x}=\mathbb{E}\left[\mathbf{x}_{s t} \mathbf{x}_{s t}^{H}\right] \in \mathcal{R}^{N \times N}$, can be expressed as

$$
\mathbf{R}_{x x}=\mathbf{R}_{s} \otimes \mathbf{R}_{t}
$$

where $\otimes$ denotes the Kronecker product, and $\mathbf{R}_{s} \in$ $\mathcal{R}^{N_{s} \times N_{s}}$ and $\mathbf{R}_{t} \in \mathcal{R}^{N_{t} \times N_{t}}$ are the correlation matrices in the space domain and time domain, respectively. The space domain correlation matrix, $\mathbf{R}_{s}$, has the form of a symmetric Toeplitz matrix with the first row and first column being $\mathbf{r}_{s}=\left[1, \rho_{s}^{d}, \ldots, \rho_{s}^{\left(N_{s}-1\right) d}\right]^{T}$. Similarly, the time domain correlation matrix, $\mathbf{R}_{t}$, is a symmetric Toeplitz matrix with the first row and first column being $\mathbf{r}_{t}=$ $\left[1, \rho_{t}^{T_{s}}, \ldots, \rho_{t}^{\left(N_{t}-1\right) T_{s}}\right]^{T}$. The matrix, $\mathbf{R}_{x x}$, has the form of a Toeplitz-block-Toeplitz (TBT) matrix [18], i.e., $\mathbf{R}_{x x}$ is a block Toeplitz matrix, and each sub-matrix is also a Toeplitz matrix.

\subsection{MMSE estimation of the discrete samples}

For the MMSE estimation described in (6), the optimum $\mathbf{W}_{x}$ that minimizes the MSE, $\sigma_{s t, N}^{2}$, can be found through the orthogonal principal, $\mathbb{E}\left[\left(\hat{\boldsymbol{x}}_{s t}-\mathbf{x}_{s t}\right) \mathbf{y}^{H}\right]=\mathbf{0}$. The result is

$$
\begin{aligned}
\mathbf{W}_{x}^{H}= & \sqrt{\frac{E_{n}}{1+\sigma_{w}^{2}}} \mathbf{R}_{x x} \mathbf{H}^{H} \\
& \times\left[\frac{E_{n}}{1+\sigma_{w}^{2}} \mathbf{H R}_{x x} \mathbf{H}^{H}+\frac{E_{n} \sigma_{w}^{2}}{1+\sigma_{w}^{2}} \mathbf{H} \mathbf{H}^{H}+\sigma_{z}^{2} \mathbf{I}_{N}\right]^{-1},
\end{aligned}
$$


The conditional error correlation matrix, $\mathbf{R}_{e e \mid \mathbf{H}}^{(x)}=$ $\mathbb{E}\left[\mathbf{e}_{s} \mathbf{e}_{s}^{H} \mid \mathbf{H}\right]$, with $\mathbf{e}_{s}=\hat{\mathbf{x}}_{s t}-\mathbf{x}_{s t}$, can then be calculated as

$$
\begin{aligned}
\mathbf{R}_{e e \mid \mathbf{H}}^{(x)}= & \mathbf{R}_{x x}-\mathbf{R}_{x x} \mathbf{H}^{H} \\
& \times\left[\mathbf{H} \mathbf{R}_{x x} \mathbf{H}^{H}+\sigma_{w}^{2} \mathbf{H} \mathbf{H}^{H}+\left(1+\sigma_{w}^{2}\right) \frac{\theta \delta}{\gamma_{0}} \mathbf{I}_{N}\right]^{-1} \mathbf{H R}_{x x} \\
= & {\left[\mathbf{R}_{x x}^{-1}+\frac{\mathbf{H} \mathbf{H}^{H}}{\sigma_{w}^{2} \mathbf{H} \mathbf{H}^{H}+\left(1+\sigma_{w}^{2}\right) \frac{\theta \delta}{\gamma_{0}} \mathbf{I}_{N}}\right]^{-1}, }
\end{aligned}
$$

where the orthogonal principal is used in the first equality, and the second equality is based on the identity $\mathbf{D}^{-1}+$ $\mathbf{D}^{-1} \mathbf{C}\left(\mathbf{A}-\mathbf{B D}^{-1} \mathbf{C}\right)^{-1} \mathbf{B D}^{-1}=\left(\mathbf{D}-\mathbf{C A}^{-1} \mathbf{B}\right)^{-1}$. The MSE can then be calculated as

$$
\sigma_{s t, N}^{2}=\frac{1}{N} \mathbb{E}_{\mathbf{H}}\left[\operatorname{trace}\left(\mathbf{R}_{e e \mid \mathbf{H}}^{(x)}\right)\right]
$$

where trace (A) returns the trace of the matrix A.

In Equations (11) and (12), the calculation of the MSE involves matrix inversion, the trace operation, and the expectation operation. The value of the MSE can be evaluated numerically. In order to explicitly identify the impacts of the node density and sampling rate on the MSE, we will first focus on the analysis of system operating in the AWGN channel, and this will allow us to express the MSE as a closed form expression of the node density and sampling rate. The MSE obtained under the AWGN channel will be compared to the MSE under the fading environment later in this section.

Proposition 1. When $N_{s} \rightarrow \infty$ and $N_{t} \rightarrow \infty$ while keeping both $N_{t} \rightarrow \infty$ and $\theta$ finite, the MSE of the estimation of the discrete samples collected by the sensors and transmitted in an AWGN channel is

$$
\sigma_{s t}^{2}=\lim _{N \rightarrow \infty} \sigma_{s t, N}^{2}=\frac{\sqrt{2}}{\pi \sqrt{\beta}} \cdot K\left(\sqrt{\frac{\alpha}{\beta}}\right)
$$

where $K(\cdot)$ is the complete elliptic integral of the first kind ([19], Equation (8.112.1)), and

$$
\begin{aligned}
\alpha= & \frac{8}{\sigma_{w}^{2}+\left(1+\sigma_{w}^{2}\right) \frac{\theta \delta}{\gamma_{0}}} \cdot \frac{\rho_{s}^{\frac{1}{\delta}}}{1-\rho_{s}^{\frac{2}{\delta}}} \cdot \frac{\rho_{t}^{\frac{1}{\theta}}}{1-\rho_{t}^{\frac{2}{\theta}}}, \\
\beta= & \frac{1}{2}+\frac{1}{\sigma_{w}^{2}+\left(1+\sigma_{w}^{2}\right) \frac{\theta \delta}{\gamma_{0}}} \cdot\left(1+\frac{2 \rho_{s}^{\frac{2}{\delta}}}{1-\rho_{s}^{\frac{2}{\delta}}}\right)\left(1+\frac{2 \rho_{t}^{\frac{2}{\theta}}}{1-\rho_{t}^{\frac{2}{\theta}}}\right) \\
& +\frac{1}{2}\left[\frac{1}{\sigma_{w}^{2}+\left(1+\sigma_{w}^{2}\right) \frac{\theta \delta}{\gamma_{0}}}\right]^{2}+\frac{\alpha}{2} .
\end{aligned}
$$

Proof. The proof is given in Appendix 1.
In Proposition 1, the spatial-temporal sampling affects the MSE in the form of the following functions, $f_{1}\left(\rho_{s}, \delta\right)=$ $\frac{\rho_{s}^{\frac{2}{\delta}}}{1-\rho_{s}^{\frac{2}{\delta}}}, g_{1}\left(\rho_{s}, \delta\right)=\frac{\rho_{s}^{\frac{1}{\delta}}}{1-\rho_{s}^{\frac{2}{\delta}}}, f_{1}\left(\rho_{t}, \theta\right)=\frac{\rho_{t}^{\frac{2}{\theta}}}{1-\rho_{t}^{\frac{2}{\theta}}}, g_{1}\left(\rho_{t}, \theta\right)=$ $\frac{\rho_{t}^{\frac{1}{\theta}}}{1-\rho_{t}^{\frac{2}{\theta}}}$, and $f_{2}(\delta, \theta)=\frac{1}{\sigma_{w}^{2}+\left(1+\sigma_{w}^{2}\right) \frac{\theta \delta}{\gamma_{0}}}$. Among them, $f_{1}\left(\rho_{s}, \delta\right)$ and $g_{1}\left(\rho_{s}, \delta\right)$ are related to the spatial correlation, and they are increasing functions of $\delta . f_{1}\left(\rho_{t}, \theta\right)$ and $g_{1}\left(\rho_{t}, \theta\right)$ are related to the temporal correlation, and they are increasing functions of $\theta$. The function $f_{2}(\delta, \theta)$ is a decreasing function of both $\delta$ and $\theta$.

In Proposition 1, if we assume that the data is spatially correlated but temporally uncorrelated, then the MSE of the spatial samples can be simplified as follows.

Corollary 1. If $\rho_{t}=0$, the asymptotic MSE of the estimation for the spatially correlated samples is

$$
\begin{aligned}
\sigma_{s}^{2}= & {\left[\left(1+\frac{1}{\sigma_{w}^{2}+\left(1+\sigma_{w}^{2}\right) \frac{\theta \delta}{\gamma_{0}}}\right)^{2}\right.} \\
& \left.+\frac{4 \rho_{s}^{\frac{2}{\delta}}}{\left(\sigma_{w}^{2}+\left(1+\sigma_{w}^{2}\right) \frac{\theta \delta}{\gamma_{0}}\right)\left(1-\rho_{s}^{\frac{2}{\delta}}\right)}\right]^{-\frac{1}{2}} .
\end{aligned}
$$

Proof. Setting $\rho_{t}=0$ leads to $\alpha=0$ and $\beta=$ $0.5+\left[1+2 f_{1}\left(\rho_{s}, \delta\right)\right] f_{2}(\delta, \theta)+0.5 f_{2}(\delta, \theta)^{2}$. Equation (15) can be obtained by substituting $\beta$ into (13).

When $\sigma_{w}^{2}=0$, the result in Corollary 1 coincides with ([5], [Equation (12)]), where only the spatial samples are considered. It was shown in [5] analytically that $\sigma_{s}^{2}$ is an increasing function in $\delta$.

Similarly, based on the symmetry between the space and time domains, we can get the MSE of the estimation of the temporal samples for a given node, by exchanging $\rho_{s}$ with $\rho_{t}$, and $\delta$ with $\theta$ in (15).

Figure 2 shows the asymptotic MSE as a function of the spatial node density, $\delta$, under various values of the correlation coefficients, $\rho_{t}$ and $\rho_{s}$, in an AWGN channel with SNR $\gamma_{0}=10 \mathrm{~dB}$. Define $\gamma_{w}=\frac{P_{0}}{\sigma_{w}^{2}}$ as the measurement SNR per unit area. The temporal sampling rate is $\theta=10 \mathrm{sample} / \mathrm{sec}$. Data samples are assumed to be a zero-mean Gaussian process with the auto-correlation function given in (1). The simulation results are obtained by using $N_{s}=N_{t}=60$ samples to approximate infinite number of samples. Excellent match is observed between the simulation results with finite number of samples and the asymptotic results with infinite number of samples. As expected, the MSE performance improves as $\gamma_{w}$ increases. When $\gamma_{w}=10 \mathrm{~dB}$, there is only a slight difference between 


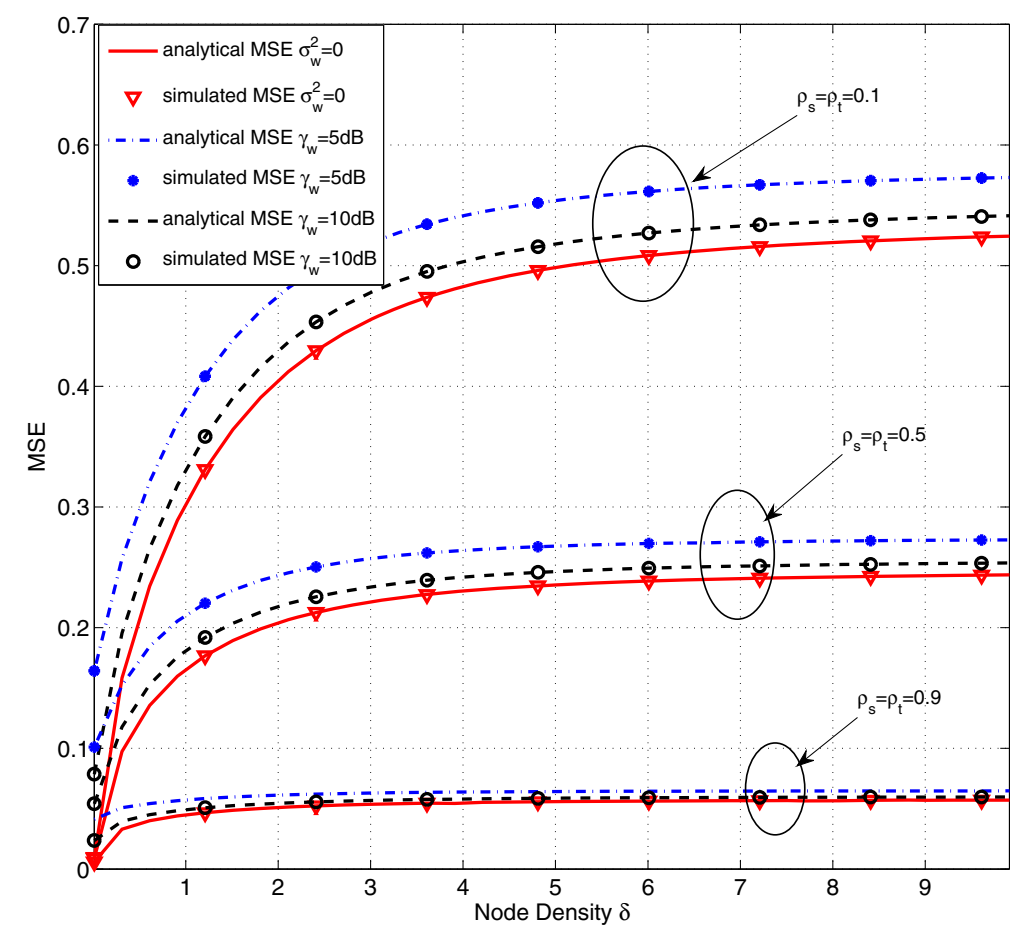

Figure 2 The asymptotic MSE of the estimated discrete data samples in the 1-D network under various values of measurement SNR $\gamma_{w}$ (AWGN, $\gamma_{0}=10 \mathrm{~dB}, \theta=10 \mathrm{~Hz}$ ).

the system with and without measurement noise. In addition, the MSE is an increasing function in the node density for all configurations. This indicates that the MSE for the discrete data samples can benefit from a smaller spatial node density. Therefore, if we only want to obtain the data at some discrete locations, we should use a node density that is as small as allowed by the application, i.e., placing exactly one sensor at each desired measurement location will obtain the optimum performance. Due to the symmetry between the space and time domain, the above analysis is also true for the relationship between $\sigma_{s t}^{2}$ and $\theta$. In addition, the MSE approaches a constant as $\delta \rightarrow \infty$. The result is corroborated by the following corollary.

Corollary 2. For the estimation of the discrete samples collected by the sensors and transmitted in AWGN channels, given a sampling rate $\theta$, when $\delta \rightarrow \infty$, the asymptotic MSE approaches a constant as

$$
\lim _{\delta \rightarrow \infty} \sigma_{s t}^{2}=\frac{2}{\pi}\left[1-\frac{2 \gamma_{0}}{\left(1+\sigma_{w}^{2}\right) \theta \log \left(\rho_{s}\right)} \cdot \frac{1+\rho_{t}^{\frac{1}{\theta}}}{1-\rho_{t}^{\frac{1}{\theta}}}\right]^{-\frac{1}{2}} \cdot K\left(\Delta_{\delta}\right),
$$

with $\Delta_{\delta}=\left[\frac{8 \gamma_{0} \rho_{t}^{\frac{1}{\theta}}}{2 \gamma_{0}\left(1+\rho_{t}^{\frac{1}{\theta}}\right)^{2}-\left(1+\sigma_{w}^{2}\right) \theta \log \left(\rho_{s}\right)\left(1-\rho_{t}^{\frac{2}{\theta}}\right)}\right]^{\frac{1}{2}}$.

Proof. The proof is in Appendix 2.
Corollary 3. For the estimation of the discrete samples collected by the sensors and transmitted in AWGN channels, when both $\theta \rightarrow \infty$ and $\delta \rightarrow \infty$, we have

$$
\lim _{\delta \rightarrow \infty, \theta \rightarrow \infty} \sigma_{s t}^{2}=\frac{2}{\pi}\left(1+\frac{4}{\varpi}\right)^{-\frac{1}{2}} \cdot K\left(\sqrt{\frac{4}{4+\varpi}}\right),
$$

where $\varpi=\frac{\log \left(\rho_{s}\right) \log \left(\rho_{t}\right)\left(1+\sigma_{w}^{2}\right)}{\gamma_{0}}$.

Proof. Equation (17) can be directly proved by substituting $\lim _{\theta \rightarrow \infty} \theta\left(1-\rho_{t}^{\frac{1}{\theta}}\right)=-\log \left(\rho_{t}\right)$ into (45).

In (17), when both $\theta$ and $\delta$ tend infinity, the limit depends on the correlation coefficients and the SNR. The relationship between the limit and $\rho_{s}, \rho_{t}, \gamma_{0}$ is given by the following corollary.

Corollary 4. The limit in Corollary 3 is proportional to $\rho_{s}$ and $\rho_{t}$, and inversely proportional to the SNR $\gamma_{0}$.

Proof. The proof is in Appendix 3.

We next compare in Figure 3 the MSE for systems operating in AWGN channels and fading channels, respectively. The MSE in fading channels is obtained with a 


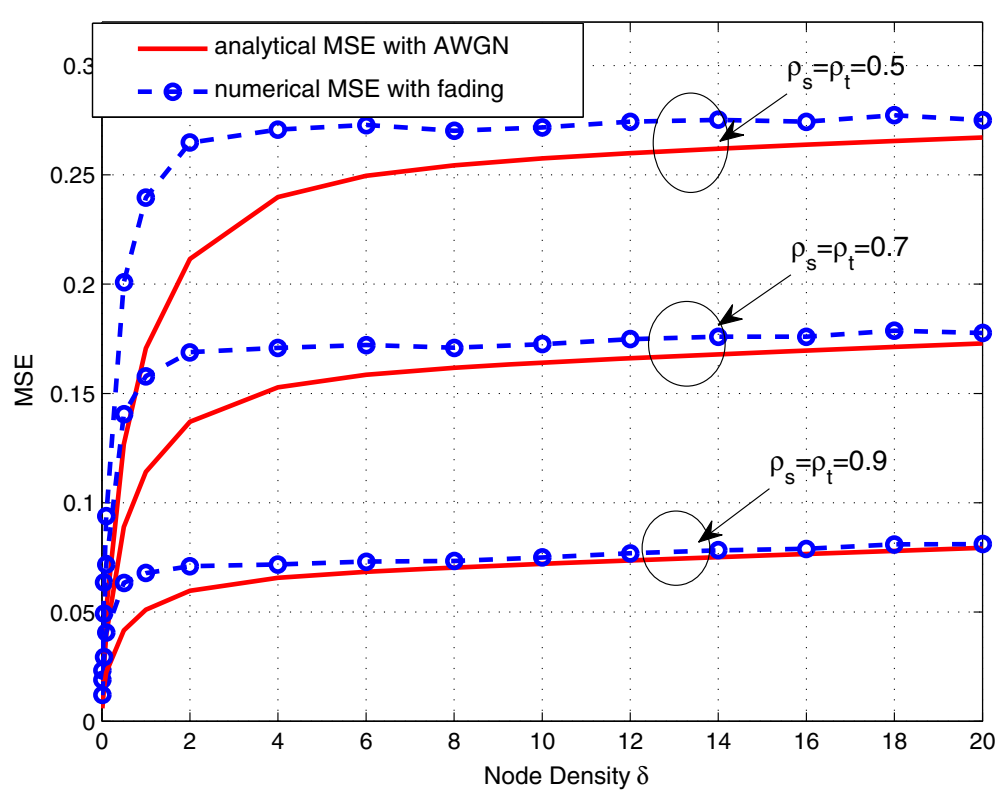

Figure 3 Impacts of fading on the asymptotic MSE of the estimated discrete data samples in 1-D networks $\left(\gamma_{0}=10 \mathrm{~dB}, \theta=10 \mathrm{~Hz}, \sigma_{w}^{2}=0\right)$.

hybrid analytical and simulation method, i.e., given $\mathbf{H}$, the conditional MSE can be calculated by performing the trace operation over (11), and the unconditional MSE can then be obtained by averaging over a large number of independent implementations of $\mathbf{H}$. The parameters, $\gamma_{0}$ and $\theta$, are the same as those in Figure 2, and the variance of the measurement noise is $\sigma_{w}^{2}=0$. The fading MSE is lower bounded by its AWGN counterpart. The difference between the MSE of these two types of networks gradually diminishes as $\rho_{s}$ and $\rho_{t}$ increases. When $\rho_{s}=\rho_{t}=0.9$, there is only a slight difference between the two, especially when the node density is high. In addition, both of the two networks have the same performance trend, i.e., the MSE is an increasing function in $\delta$. Therefore, the analytical result in AWGN channel can provide a rough guideline on the design of systems with fading.

\subsection{MMSE spatial-temporal interpolation}

This section discusses the distortion performance of space-time interpolation, i.e., the estimation of any arbitrary point on the space-time plane by interpolating the $N$ discrete space-time samples.

Since we are interested in the reconstruction fidelity of the entire space-time hyperplane, the worst case scenario is considered by estimating the data located in the middle of the square formed by four neighboring samples, as shown in Figure 4a, with the data points to be estimated being $x_{i j}^{\prime}=x\left[\left(i-\frac{1}{2}\right) d,\left(j-\frac{1}{2}\right) T_{s}\right]$, for $i=$ $1, \ldots, N_{s}$ and $j=1, \ldots, N_{t}$. Define the interpolation data vector as $\mathbf{x}_{d t}=\left[\mathbf{x}_{1}^{\prime}, \ldots, \mathbf{x}_{N_{s}}^{\prime T}\right]^{T} \in \mathcal{R}^{N \times 1}$, where $\mathbf{x}_{i}^{\prime}=$ $\left[x_{i 1}^{\prime}, x_{i 2}^{\prime}, \ldots, x_{i N_{t}}^{\prime}\right]^{T} \in \mathcal{R}^{N_{t} \times 1}$.

Based on the orthogonal principal, $\mathbb{E}\left[\left(\hat{\mathbf{x}}_{d t}-\mathbf{x}_{d t}\right) \hat{\mathbf{x}}_{s t}^{H}\right]=$ $\mathbf{0}$, where $\hat{\mathbf{x}}_{d t}$ is an estimate of $\mathbf{x}_{d t}$, the MMSE space-time interpolations can be expressed by

$$
\hat{\mathbf{x}}_{d t}=\mathbf{R}_{d \hat{x}} \mathbf{R}_{\hat{x} \hat{x}}^{-1} \hat{\mathbf{x}}_{s t},
$$

where

$$
\begin{aligned}
\mathbf{R}_{d \hat{x}} \triangleq \mathbb{E}\left(\mathbf{x}_{d t} \hat{\mathbf{x}}_{s t}^{H}\right)= & \sqrt{\frac{E_{n}}{1+\sigma_{w}^{2}}} \mathbf{R}_{d x} \mathbf{H}^{H} \mathbf{W}_{x}, \\
\mathbf{R}_{\hat{x} \hat{x}} \triangleq \mathbb{E}\left(\hat{\mathbf{x}}_{s t} \hat{\mathbf{x}}_{s t}^{H}\right)= & \mathbf{W}_{x}^{H}\left(\frac{E_{n}}{1+\sigma_{w}^{2}} \mathbf{H R}_{x x} \mathbf{H}^{H}\right. \\
& \left.+\frac{E_{n} \sigma_{w}^{2}}{1+\sigma_{w}^{2}} \mathbf{H H}^{H}+\sigma_{z}^{2} \mathbf{I}_{N}\right) \mathbf{W}_{x},
\end{aligned}
$$

with $\mathbf{R}_{d x} \triangleq \mathbb{E}\left(\mathbf{x}_{d t} \mathbf{x}_{s t}^{H}\right)=\mathbf{R}_{s}^{\prime} \otimes \mathbf{R}_{t}^{\prime}$ being a TBT matrix. The matrix $\mathbf{R}_{s}^{\prime}$ is a Toeplitz matrix with the first row being $\rho_{s}^{\frac{d}{2}}\left[1,1, \rho_{s}^{d}, \ldots, \rho_{s}^{\left(N_{s}-2\right) d \mid}\right]^{T} \in \mathcal{R}^{N_{s} \times 1}$, and the first column $\rho_{s}^{\frac{d}{2}}\left[1, \rho_{s}^{d}, \ldots, \rho_{s}^{\left(N_{s}-1\right) d}\right]^{T} \in \mathcal{R}^{N_{s} \times 1}$. Similarly $\mathbf{R}_{t}^{\prime}$ is a Toeplitz matrix with the first row being $\rho_{t}^{\frac{T_{s}}{2}}\left[1,1, \rho_{t}^{T_{s}}, \ldots, \rho_{t}^{\left(N_{t}-2\right) T_{s}}\right]^{T} \in \mathcal{R}^{N_{t} \times 1}$, and the first 



Figure 4 Three types of interpolations for the 1-D network. (a) Space-time interpolation.

(b) Space interpolation. (c) Time interpolation.

column $\rho_{t}^{\frac{T_{s}}{2}}\left[1, \rho_{t}^{T_{s}}, \ldots, \rho_{t}^{\left(N_{t}-1\right) T_{s}}\right]^{T} \in \mathcal{R}^{N_{t} \times 1}$. Combining (18) with (19), we have

$$
\begin{aligned}
\hat{\mathbf{x}}_{d t}= & \sqrt{\frac{E_{n}}{1+\sigma_{w}^{2}}} \mathbf{R}_{d x} \mathbf{H}^{H} \\
& \times\left[\frac{E_{n}}{1+\sigma_{w}^{2}} \mathbf{H} \mathbf{R}_{x x} \mathbf{H}^{H}+\frac{E_{n} \sigma_{w}^{2}}{1+\sigma_{w}^{2}} \mathbf{H} \mathbf{H}^{H}+\sigma_{z}^{2} \mathbf{I}_{N}\right]^{-1} \mathbf{y} .
\end{aligned}
$$

The corresponding error correlation matrix, $\mathbf{R}_{e e}^{(d)} \triangleq$ $\mathbb{E}\left[\left(\hat{\mathbf{x}}_{d t}-\mathbf{x}_{d t}\right)\left(\hat{\mathbf{x}}_{d t}-\mathbf{x}_{d t}\right)^{H}\right]$, can then be calculated by

$$
\begin{aligned}
\mathbf{R}_{e e}^{(d)}=\mathbb{E}_{\mathbf{H}}\{ & \mathbf{R}_{x x}-\mathbf{R}_{d x} \mathbf{H}^{H}\left[\mathbf{H} \mathbf{R}_{x x} \mathbf{H}^{H}+\sigma_{w}^{2} \mathbf{H} \mathbf{H}^{H}\right. \\
& \left.\left.+\left(1+\sigma_{w}^{2}\right) \frac{\theta \delta}{\gamma_{0}} \mathbf{I}_{N}\right]^{-1} \mathbf{H} \mathbf{R}_{x d}\right\}
\end{aligned}
$$

where $\mathbf{R}_{d d}=\mathbb{E}\left(\mathbf{x}_{d t} \mathbf{x}_{d t}^{H}\right)=\mathbf{R}_{x x}$ is used in the above equation, and $\mathbf{R}_{x d}=\mathbf{R}_{d x}^{H}$.

The MSE for the space-time interpolation when operating in a fading channel can be evaluated numerically by performing the trace operation over (21). To gain more insights on the impacts of node density and sampling rate, we next perform asymptotic analysis for systems operating in AWGN channels.

Proposition 2. When $N_{s} \rightarrow \infty$ and $N_{t} \rightarrow \infty$ while keeping both $\delta$ and $\theta$ finite, the MSE of the spatialtemporal interpolation for a network operating in AWGN channels is

$$
\begin{aligned}
\vartheta_{s t}^{2} \triangleq \lim _{N \rightarrow \infty} \vartheta_{s t, N}^{2}=\frac{1-\rho_{t}^{\frac{1}{\theta}}}{1+\rho_{t}^{\frac{1}{\theta}}} & \left\{1+\int_{-\frac{1}{2}}^{\frac{1}{2}} \frac{1+\cos (2 \pi f)}{v-\cos (2 \pi f)}\right. \\
\cdot & {\left.\left[\frac{q-\cos (2 \pi f)}{p-\cos (2 \pi f)}\right]^{\frac{1}{2}} d f\right\} }
\end{aligned}
$$

where

$$
\begin{aligned}
& v=\frac{1+\rho_{t}^{\frac{2}{\theta}}}{2 \rho_{t}^{\frac{1}{\theta}}}, p=v+\frac{1}{2\left(\sigma_{w}^{2}+\left(1+\sigma_{w}^{2}\right) \frac{\theta \delta}{\gamma_{0}}\right)} \cdot \frac{1-\rho_{t}^{\frac{2}{\theta}}}{\rho_{t}^{\frac{1}{\theta}}} \cdot \frac{1+\rho_{s}^{\frac{1}{\delta}}}{1-\rho_{s}^{\frac{1}{\delta}}}, \\
& q=v+\frac{1}{2\left(\sigma_{w}^{2}+\left(1+\sigma_{w}^{2}\right) \frac{\theta \delta}{\gamma_{0}}\right)} \cdot \frac{1-\rho_{t}^{\frac{2}{\theta}}}{\rho_{t}^{\frac{1}{\theta}}} \cdot \frac{1-\rho_{s}^{\frac{1}{\delta}}}{1+\rho_{s}^{\frac{1}{\delta}}} .
\end{aligned}
$$

Proof. The proof is in Appendix 4.

The results in Proposition 2 illustrate the asymptotic MSE performance for the MMSE interpolation in both the space and time domains. Even though the MSE in Proposition 2 is expressed as an explicit function of the correlation coefficients and the space-time sample rates, it is expressed in the form of an integral and eludes a closed-form expression. It should be noted that the integrand is composed for elementary functions, and the integration limit is finite. Therefore the integral can be easily evaluated numerically. To gain further insight on the impact of the space-time correlation on the estimation performance, we consider in the following section the interpolation in just one domain.

\subsection{Interpolation in the space or time domain}

In this section, we consider the MSE performance of interpolation in the space domain as in Figure $4 \mathrm{~b}$ or in the time domain as in Figure 4c, but not both. Studying the interpolation in one domain will help quantify the impact of node density or sampling rate on the estimation MSE. The analytical asymptotic study is performed for systems operating in AWGN channels.

Due to the symmetry between the space and time domains, it is sufficient to study the interpolation in the space domain. From Figure $4 b$, the coordinates of the data to be estimated during the spatial interpolation are $\left[\left(i+\frac{1}{2}\right) d, j T_{s}\right]$, for $i=0, \ldots, N_{s}-1$ and $j=0, \ldots, N_{t}-1$. The asymptotic MSE of the spatial interpolation is given in the following proposition. 
Proposition 3. When $N_{t} \rightarrow \infty$ and $N_{s} \rightarrow \infty$, while keeping $\delta$ and $\theta$ finite, the MSE of the estimated data during the spatial interpolation for a network operating in AWGN channels is

$$
\begin{aligned}
\vartheta_{s}^{2}= & \frac{2}{\pi} \cdot\left[\sigma_{w}^{2}+\left(1+\sigma_{w}^{2}\right) \frac{\theta \delta}{\gamma_{0}}\right] \cdot \frac{1-\rho_{s}^{\frac{1}{\delta}}}{1+\rho_{s}^{\frac{1}{\delta}}} \cdot \frac{1}{\sqrt{(p-1)(q+1)}} \\
& \cdot\left[(p-q) K\left(\alpha_{1}\right)+\frac{(q-v)(p+1)}{v+1} \Pi\left(\beta_{1}, \alpha_{1}\right)\right]
\end{aligned}
$$

where $v, p, q$ are defined in (23),

$$
\alpha_{1}=\left[\frac{2(p-q)}{(p-1)(q+1)}\right]^{\frac{1}{2}}, \beta_{1}=\frac{2(p-v)}{(p-1)(v+1)},
$$

and $\Pi(\cdot)$ is the complete elliptic integral of the third kind [19].

\section{Proof. The proof is in Appendix 5.}

If we assume the data samples are temporally uncorrelated $\left(\rho_{t}=0\right)$, and perform spatial interpolation based on the spatially correlated but temporally uncorrelated data samples, then the MSE given in Proposition 3 can be simplified as follows.

Corollary 5. If $\rho_{t}=0$, the asymptotic MSE of the estimation for the spatial interpolation is

$$
\begin{aligned}
\vartheta_{s}^{2}= & {\left[\sigma_{w}^{2}+\left(1+\sigma_{w}^{2}\right) \frac{\delta \theta}{\gamma_{0}}+\frac{1-\rho_{s}^{\frac{1}{\delta}}}{1+\rho_{s}^{\frac{1}{\delta}}}\right]^{\frac{1}{2}} } \\
& \times\left[\sigma_{w}^{2}+\left(1+\sigma_{w}^{2}\right) \frac{\delta \theta}{\gamma_{0}}+\frac{1+\rho_{s}^{\frac{1}{\delta}}}{1-\rho_{s}^{\frac{1}{\delta}}}\right]^{-\frac{1}{2}}
\end{aligned}
$$

Proof. When $\rho_{t}=0$, we have $\Lambda\left(\rho_{t}^{T_{s}}, f_{2}\right)=1$. Substituting $\Lambda\left(\rho_{t}^{T_{s}}, f_{2}\right)=1$ into (52) directly leads to (26).

When $\sigma_{w}^{2}=0$, the result in Corollary 5 simplifies to ([6], Proposition 2), where only the spatial data correlation is considered. It was proven in [6] that the MSE in (26) is a decreasing function of the node density $\delta$.

Figure 5 compares the asymptotic MSE performance between the spatial interpolation and the space-time interpolation. In the simulation, $\rho_{t}=0.1$ and $\sigma_{w}^{2}=0$ and all other parameters are the same as those in Figure 2. As expected, performing interpolation in the space domain alone leads to a better performance compared to interpolation in both the space and time domains. The difference increases as the spatial correlation coefficient, $\rho_{s}$, increases. Different from the results in Figure 2, it is observed that the MSE of the spatial interpolation or space-time interpolation is a decreasing function of the

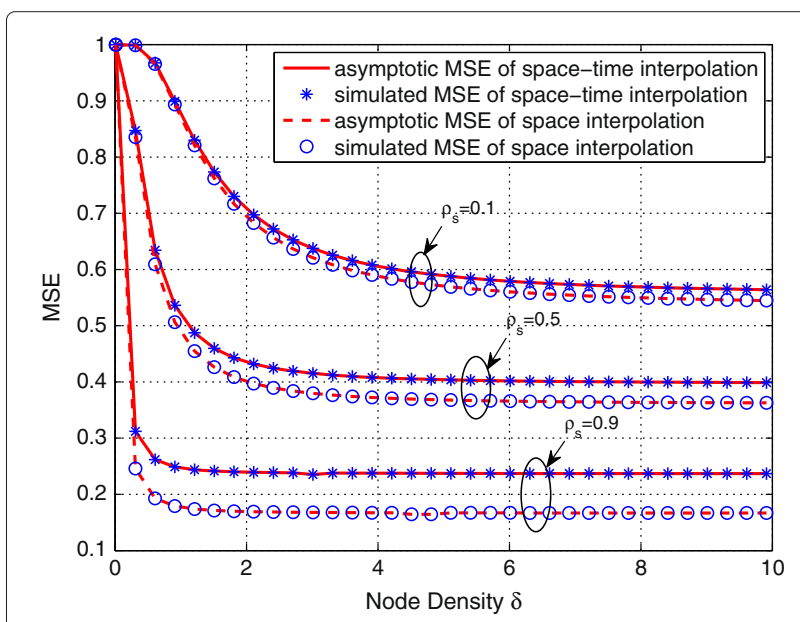

Figure 5 The asymptotic MSE of space-time interpolation and space interpolation in the 1-D network (AWGN, $\gamma_{0}=10 \mathrm{~dB}$, $\sigma_{w}^{2}=0, \rho_{t}=0.1, \theta=10 \mathrm{~Hz}$ ).

spatial node density $\delta$. This can be intuitively explained by the fact that the spatial interpolation depends mainly on the spatial correlation among the sensor nodes, and a higher node density means a stronger spatial correlation among the data samples, thus a better estimation fidelity.

It can be seen from Figure 5 that, when $\delta \rightarrow \infty$, the MSE approaches a lower bound, which is stated in the following corollary.

Corollary 6. The following relationship holds for the MSE of the estimation for the data samples $\sigma_{s t}^{2}$ and the MSE of the spatial interpolation $\vartheta_{s}^{2}$

$$
\begin{aligned}
\lim _{\delta \rightarrow \infty} \vartheta_{s}^{2} & =\left[\frac{2}{\pi}\left(1-\frac{2 \gamma_{0}}{\left(1+\sigma_{w}^{2}\right) \theta \log \left(\rho_{s}\right)} \cdot \frac{1+\rho_{t}^{\frac{1}{\theta}}}{1-\rho_{t}^{\frac{1}{\theta}}}\right)^{-\frac{1}{2}} \cdot K\left(\Delta_{\delta}\right)\right] \\
& \geq \lim _{\delta \rightarrow \infty} \sigma_{s t}^{2},
\end{aligned}
$$

with $\Delta_{\delta}$ defined in Corollary 2.

Proof. The proof is in Appendix 6.

Due to the symmetry between the space and the time domains, we can get the MSE of the time interpolation, as shown in Figure 4c, by exchanging $\rho_{s}$ with $\rho_{t}$, and $\delta$ with $\theta$ in Proposition 3, and Corollaries 5 and 6.

\subsection{Optimum spatial-temporal sampling}

It can be seen from Figure 5 that, when $\delta$ is small, the MSE decreases dramatically as $\delta$ increases. When $\delta$ reaches a certain threshold, no apparent performance gain can be achieved by increasing $\delta$ further, i.e., the slope of $\vartheta_{s t}^{2}$ 
approaches zero as $\delta$ increase. The above statement is also true for the sampling rate $\theta$.

In the space domain, we can find the optimum node density, $\delta_{0}$, by solving the equation $\left|\frac{\partial \vartheta_{s t}^{2}}{\partial \delta}\right|_{\delta_{0}}=\epsilon_{s}$, with $\epsilon_{s}$ being a small number. Figure 6 shows the optimum node density in AWGN channels as a function of the spatial correlation coefficient $\rho_{s}$, under various values of the sampling rate $\theta$. The parameters are $\rho_{t}=0.5, \sigma_{w}^{2}=0$, and $\epsilon_{s}=10^{-3}$. The results in this figure demonstrate that the optimum node density decreases almost linearly as $\rho_{s}$ increases. Therefore, for the estimation of the spatial interpolation, a smaller node density is required for a field with a stronger spatial correlation. Moreover, the optimal node density converges as the sampling rate $\theta$ increases, i.e., the optimum node densities are almost identical for $\theta=10$ and $\theta=50 \mathrm{~Hz}$. This further corroborates that increasing the sampling rate beyond a certain threshold yields negligible performance gain. Similar results are observed for the optimum sampling rate due to the space-time symmetry.

\section{Optimum node density in 2-D networks}

The impacts of spatial-temporal sampling on the estimation fidelity in a 2-D network, as shown in Figure 1b, are studied in this section. In the space domain, the $N_{s}$ sensor nodes are located on a square grid. In the time domain, each sensor collects $N_{t}$ data samples. The spacetime coordinate for the sample $x_{i k m}$ is [ $(i-1) d,(k-$ $\left.1) d,(m-1) T_{s}\right]$, for $i, k=1, \ldots, M_{s}, m=1, \ldots, N_{t}$, with $M_{s}=\sqrt{N_{s}}$. It should be noted that the spatial node density in a 2-D sensor network is $\delta=\frac{1}{d^{2}}$, which is different from the 1-D case.

Stacking all the spatial-temporally correlated data samples into a column vector, we have $\boldsymbol{\xi}_{s t}=$

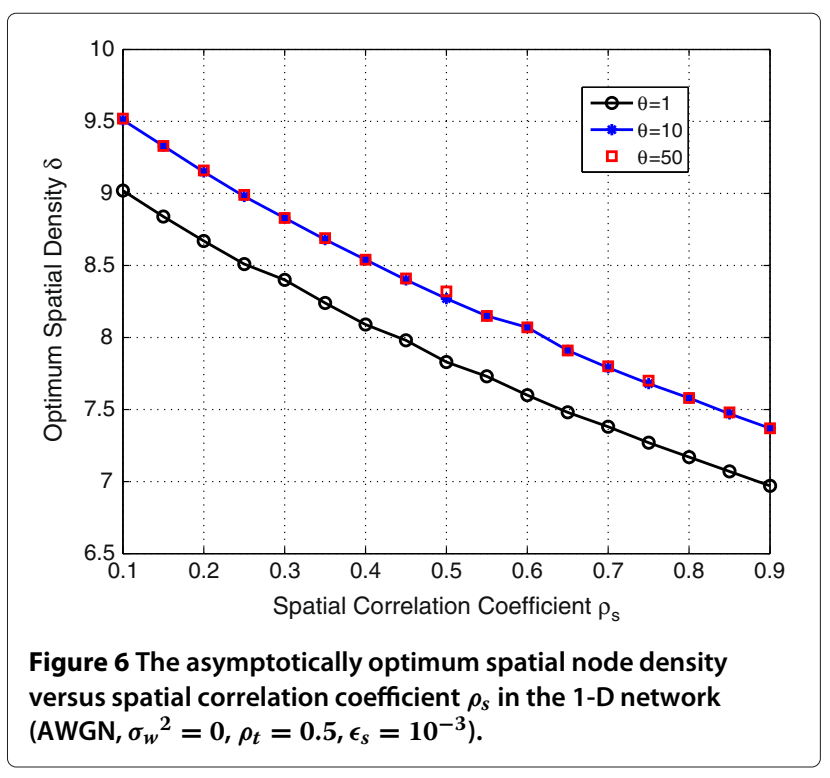

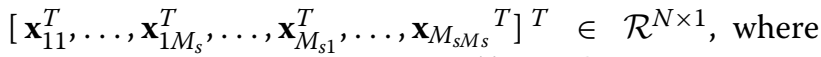
$\mathbf{x}_{m_{1} m_{2}}=\left[x_{m_{1} m_{2} 1}, \ldots, x_{m_{1} m_{2}} N_{t}\right]^{T} \in \mathcal{R}^{N_{t} \times 1}$. The autocorrelation matrix, $\boldsymbol{\Phi}_{x x}=\mathbb{E}\left[\boldsymbol{\xi}_{s t} \boldsymbol{\xi}_{s t}^{H}\right] \in \mathcal{R}^{N \times N}$, can be represented as

$$
\boldsymbol{\Phi}_{x x}=\mathbf{R}_{s s} \otimes \mathbf{R}_{t}
$$

where $\mathbf{R}_{s s} \in \mathcal{R}^{N_{s} \times N_{s}}$ and $\mathbf{R}_{t} \in \mathcal{R}^{N_{t} \times N_{t}}$ are the correlation matrices in the space domain and time domain, respectively. The matrix, $\mathbf{R}_{s s}$, assumes the form of a TBT matrix as defined in ([6], Equation (20)) for the 2-D spatially correlated network. The matrix $\mathbf{R}_{t}$ is a symmetric Toeplitz matrix as in Equation (9). Therefore, the matrix, $\boldsymbol{\Phi}_{x x}$, is a 3-level Toeplitz matrix ([20], Definition 1), i.e., $\boldsymbol{\Phi}_{x x}$ has an outermost block Toeplitz structure, and each block is still a block Toeplitz matrix, down to the innermost block with the form of an ordinary Toeplitz matrix.

Mirroring the analysis in the 1-D case, we will study, in the following two sections, the optimum spatial-temporal sampling for the MMSE estimation of the discrete data samples, and the MMSE interpolation, respectively.

\subsection{MMSE estimation of the discrete samples}

With the first-step MMSE estimation in Lemma 1, we have the MSE, $\psi_{s t, N}^{2}=\frac{1}{N} \mathbb{E}\left[\left\|\hat{\boldsymbol{\xi}}_{s t}-\boldsymbol{\xi}_{s t}\right\|^{2}\right]$, as

$$
\psi_{s t, N}^{2}=\frac{1}{N} \mathbb{E}_{\mathbf{H}}\left[\operatorname{trace}\left(\boldsymbol{\Phi}_{x x}^{-1}+\frac{\mathbf{H H}^{H}}{\sigma_{w}^{2} \mathbf{H} \mathbf{H}^{H}+\left(1+\sigma_{w}^{2}\right) \frac{\theta \delta}{\gamma_{0}} \mathbf{I}_{N}}\right)^{-1}\right] \text {, }
$$

where $\hat{\boldsymbol{\xi}}_{s t}$ is the MMSE estimate of $\boldsymbol{\xi}_{s t}$. The above MSE in a fading channel can be evaluated numerically.

Following the same procedure as in 1-D networks, we derive the explicit form of the asymptotic MSE for the system in AWGN channels.

Proposition 4. When $N_{s} \rightarrow \infty$ and $N_{t} \rightarrow \infty$, while keeping $\delta$ and $\theta$ finite, the asymptotic MSE of the discrete space-time samples in a 2-D network transmitted through AWGN channels is

$$
\begin{aligned}
\psi_{s t}^{2} \triangleq \lim _{N \rightarrow \infty} \psi_{s t, N}^{2}= & \int_{-\frac{1}{2}}^{\frac{1}{2}} \int_{-\frac{1}{2}}^{\frac{1}{2}} \int_{-\frac{1}{2}}^{\frac{1}{2}}\left[\frac{1}{\Lambda_{s s}\left(f_{1}, f_{2}\right) \Lambda\left(\rho_{t}^{T_{s}}, f_{3}\right)}\right. \\
& \left.+\frac{1}{\sigma_{w}^{2}+\left(1+\sigma_{w}^{2}\right) \frac{\theta \delta}{\gamma_{0}}}\right]^{-1} d f_{1} d f_{2} d f_{3},
\end{aligned}
$$

where $\Lambda(a, f)$ is defined in (40) in Appendix 1, and

$$
\Lambda_{s s}\left(f_{1}, f_{2}\right)=\sum_{i=-\infty}^{+\infty} \sum_{k=-\infty}^{+\infty} \rho_{s}^{\sqrt{\left(i^{2}+k^{2}\right) / \delta}} e^{-j 2 \pi\left(i f_{1}+k f_{2}\right)} .
$$

Proof. The proof is in Appendix 7. 
In Proposition 4, the impacts of the spatialtemporal sampling rate are expressed through the term, $\frac{1}{\sigma_{w}^{2}+\left(1+\sigma_{w}^{2}\right) \frac{\theta \delta}{\gamma_{0}}}$, and the 3-D DTFT, $\Lambda_{x x}^{\prime}\left(f_{1}, f_{2}, f_{3}\right)=$ $\Lambda_{s s}\left(f_{1}, f_{2}\right) \Lambda\left(\rho_{t}^{T_{s}}, f_{3}\right)$. The expression of $\psi_{s t}^{2}$ eludes a closed-form. The non-closed-form expression in (30) can be easily evaluated numerically given that the integrals are of finite limits. Even though $\Lambda_{x x}^{\prime}\left(f_{1}, f_{2}, f_{3}\right)$ is expressed as the sum of an infinite series, the value of $\rho_{s} \sqrt{\left(i^{2}+k^{2}\right) / \delta}$ decreases exponentially as $i$ and $k$ increase, thus $\Lambda_{x x}^{\prime}\left(f_{1}, f_{2}, f_{3}\right)$ can be accurately approximated with moderate limits on $i$ and $k$.

If we assume that the data are temporally uncorrelated $\left(\rho_{t}=0\right)$, then the MSE of the data samples in proposition 4 can be simplified as follows.

Corollary 7. If $\rho_{t}=0$, the asymptotic MSE of the data samples in a 2-D network with AWGN channels is

$$
\psi_{s}^{2}=\int_{-\frac{1}{2}}^{\frac{1}{2}} \int_{-\frac{1}{2}}^{\frac{1}{2}}\left[\frac{1}{\Lambda_{s s}\left(f_{1}, f_{2}\right)}+\frac{1}{\sigma_{w}^{2}+\left(1+\sigma_{w}^{2}\right) \frac{\theta \delta}{\gamma_{0}}}\right]^{-1} d f_{1} d f_{2},
$$

where $\Lambda_{s s}\left(f_{1}, f_{2}\right)$ is defined in (31).

Proof. Setting $\rho_{t}=0$ in (40) leads to $\Lambda\left(\rho_{t}^{T_{s}}, f_{3}\right)=1$. Substituting $\Lambda\left(\rho_{t}^{T_{s}}, f_{3}\right)=1$ into (30) and solving the integration with respect to $f_{3}$, we can obtain (32).
The result in Corollary 7 simplifies to ([6], Proposition 3) with $\sigma_{w}^{2}=0$, where only the spatial data correlation is considered.

The asymptotic MSE of the data samples in a 2-D network is plotted as a function of the temporal sampling rate $\theta$ in Figure 7, under various values of temporal correlation coefficient $\rho_{t}$ and measurement SNR $\gamma_{w}$. The parameters are $\rho_{s}=0.5$ and $\gamma_{0}=10 \mathrm{~dB}$. For comparison, the MSE in an 1-D network is also shown in the figure. It is interesting to note that when the measurement SNR is low $\left(\gamma_{w}=5 \mathrm{~dB}\right)$ and the time correlation is high $\left(\rho_{t}=0.9\right)$, the MSE is decreasing in $\theta$; for all other cases, the MSE is an increasing function in $\theta$. This is because if $\sigma_{w}^{2}$ is large enough, the majority of the energy is used for transmitting measurement noise. In this case, when increasing $\theta$ for data with high temporal correlation, the benefit of data correlation outweighs the loss due to less energy per sample. The performance difference between $\gamma_{w}=10 \mathrm{~dB}$ and $\sigma_{w}^{2}=0$ is very small. In addition, 2-D MSE is larger (worse) than the 1-D MSE. This can be explained by the fact that, under the same spatial node density and temporal sampling rate, each node in the 2-D network needs to cover a larger area than the node in the 1-D network, thus leads to a worse performance.

The asymptotic MSE for 2-D networks in AWGN channels is compared to that in fading channels in Figure 8. Similar to the 1-D case, the MSE with fading channels is worse than its AWGN counterpart. The networks with fading channels and AWGN channels have similar performance trend, and the performance difference between

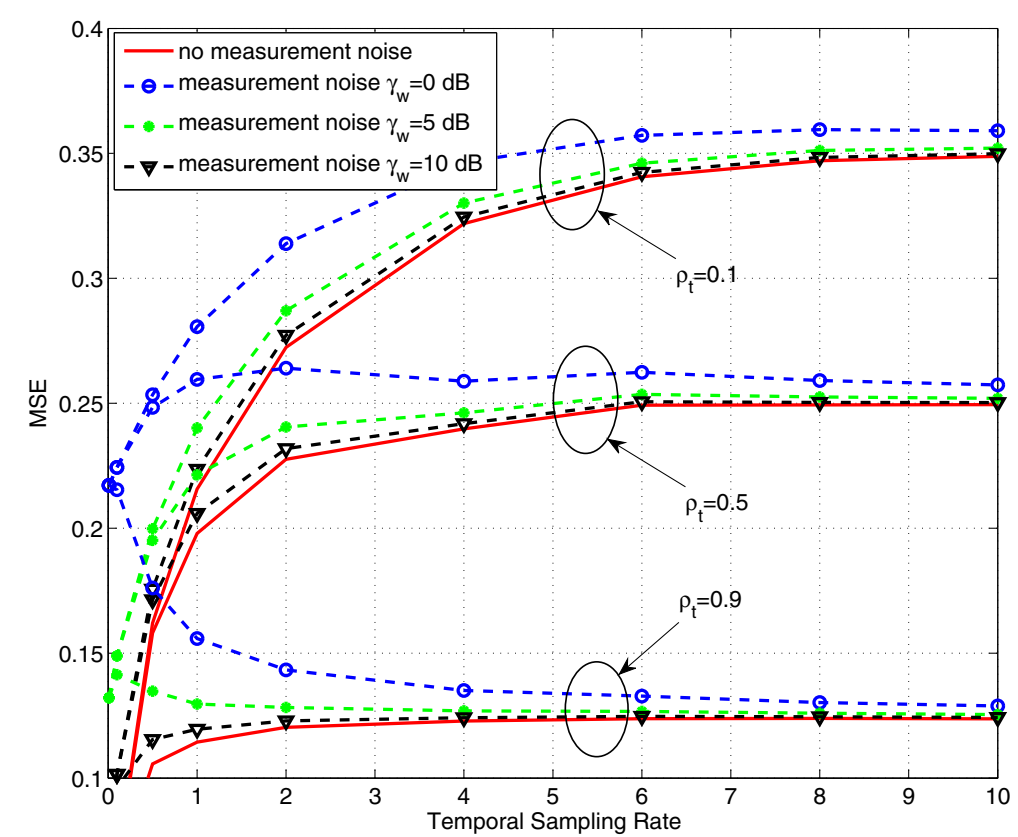

Figure 7 The MSE of the estimated discrete data samples in 2-D network (AWGN, $\gamma_{0}=10 \mathrm{~dB}, \rho_{s}=0.5$ ). 


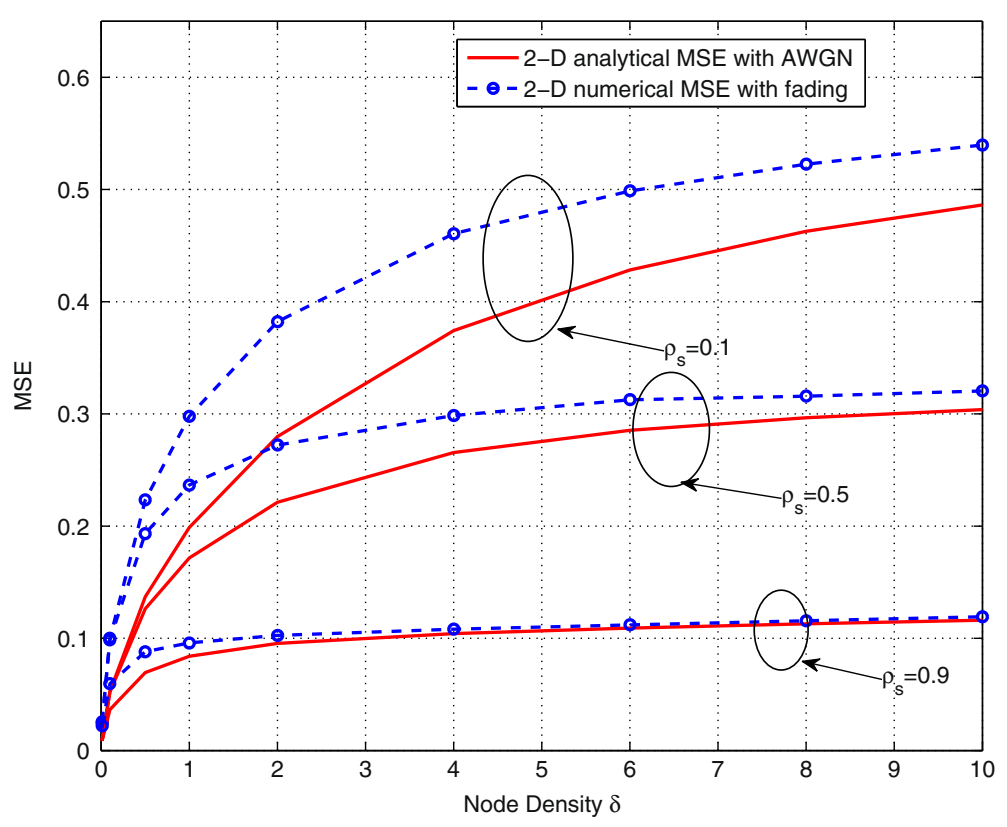

Figure 8 Impacts of fading on the MSE of the estimated discrete data samples in 2-D networks $\left(\gamma_{0}=10 \mathrm{~dB}, \theta=10 \mathrm{~Hz}, \sigma_{w}^{2}=0\right)$.

the two gradually diminishes as $\rho_{s}$ increases. When $\rho_{s}=$ 0.9 , the performance in fading and AWGN channels are almost the same at high node density.

\subsection{MMSE spatial-temporal interpolation}

The performance of spatial-temporal interpolations in a 2-D network is studied in this section. Similar to the 1$\mathrm{D}$ case, we consider the worst case by estimating the data located in the middle of the cube formed by eight adjacent data samples, with the data points to be estimated as $x_{i k m}^{\prime}=x\left[\left(i-\frac{1}{2}\right) d,\left(k-\frac{1}{2}\right) d,\left(m-\frac{1}{2}\right) T_{s}\right]$, for $i, k=1, \ldots, \sqrt{N}_{s}$ and $m=1, \ldots, N_{t}$. Correspondingly, the data vector can be expressed as $\boldsymbol{\xi}_{d t}=$ $\left[\mathbf{x}_{11}^{\prime T}, \ldots, \mathbf{x}_{1 M_{s}}^{\prime}, \ldots, \mathbf{x}_{M_{s} 1}^{\prime T}, \ldots, \mathbf{x}_{M_{s} M_{s}}^{\prime T}\right]^{T} \in \mathcal{R}^{N \times 1}$, where $\mathbf{x}_{m_{1} m_{2}}^{\prime}=\left[x_{m_{1} m_{2} 1}^{\prime}, \ldots, x_{\left.m_{1} m_{2} N_{t}\right)}^{\prime}\right]^{T} \in \mathcal{R}^{N_{t} \times 1}$.

Following the same procedure as in the 1-D case, the error correlation matrix, $\boldsymbol{\Phi}_{e e}^{(d)}=\mathbb{E}\left[\left(\hat{\boldsymbol{\xi}}_{d t}-\boldsymbol{\xi}_{d t}\right)\left(\hat{\boldsymbol{\xi}}_{d t}-\boldsymbol{\xi}_{d t}\right)^{H}\right]$, with $\hat{\boldsymbol{\xi}}_{d t}$ being the MMSE estimate of $\boldsymbol{\xi}_{d t}$, can be calculated by

$$
\begin{aligned}
\boldsymbol{\Phi}_{e e}^{(d)}= & \mathbb{E}_{\mathbf{H}}\left\{\boldsymbol{\Phi}_{x x}-\boldsymbol{\Phi}_{d x} \mathbf{H}^{H}\left[\mathbf{H} \boldsymbol{\Phi}_{x x} \mathbf{H}^{H}+\sigma_{w}^{2} \mathbf{H} \mathbf{H}^{H}\right.\right. \\
& \left.\left.+\left(1+\sigma_{w}^{2}\right) \frac{\theta \delta}{\gamma_{0}} \mathbf{I}_{N}\right]^{-1} \mathbf{H} \boldsymbol{\Phi}_{x d},\right\}
\end{aligned}
$$

where $\boldsymbol{\Phi}_{d d}=\mathbb{E}\left(\boldsymbol{\xi}_{d t} \xi_{d t}^{H}\right)=\boldsymbol{\Phi}_{x x}$ is used in the above equation. $\boldsymbol{\Phi}_{d x}=\mathbb{E}\left[\boldsymbol{\xi}_{d t} \boldsymbol{\xi}_{s t}^{H}\right]$, and $\boldsymbol{\Phi}_{x d}=\boldsymbol{\Phi}_{d x}^{H}$. The crosscorrelation matrix, $\boldsymbol{\Phi}_{d x}$, can be expressed as

$$
\boldsymbol{\Phi}_{d x}=\mathbf{R}_{d s}^{\prime} \otimes \mathbf{R}_{t}^{\prime}
$$

where $\mathbf{R}_{d s}^{\prime} \in \mathcal{R}^{N_{s} \times N_{s}}$ and $\mathbf{R}_{t}^{\prime} \in \mathcal{R}^{N_{t} \times N_{t}}$ are the crosscorrelation matrices between the data samples and the interpolations in the space domain and time domain, respectively. The matrix, $\mathbf{R}_{d s}^{\prime}$, has the form of a nonsymmetric TBT matrix as defined in ([6], Equation (27)) for the 2-D spatially correlated network. The matrix $\mathbf{R}_{t}^{\prime}$ is a Toeplitz matrix defined in Section 3.2. The matrix, $\boldsymbol{\Phi}_{d x}$, is a non-symmetric 3-level Toeplitz matrix.

For the AWGN case, the asymptotic MSE is given as follows.

Proposition 5. When $N_{s} \rightarrow \infty$ and $N_{t} \rightarrow \infty$, while keeping $\delta$ and $\theta$ finite, the asymptotic MSE of the spacetime interpolations in a 2-D network with AWGN channels is

$$
\begin{aligned}
\varphi_{s t}^{2}= & \lim _{N \rightarrow \infty} \varphi_{s t, N}^{2} \\
= & \int_{-\frac{1}{2}}^{\frac{1}{2}} \int_{-\frac{1}{2}}^{\frac{1}{2}} \int_{-\frac{1}{2}}^{\frac{1}{2}}\left[\Lambda_{x x}^{\prime}\left(f_{1}, f_{2}, f_{3}\right)\right. \\
& \left.-\frac{\left|\Lambda_{d x}^{\prime}\left(f_{1}, f_{2}, f_{3}\right)\right|^{2}}{\Lambda_{x x}^{\prime}\left(f_{1}, f_{2}, f_{3}\right)+\left(\sigma_{w}^{2}+\left(1+\sigma_{w}^{2}\right) \frac{\theta \delta}{\gamma_{0}}\right)}\right] d f_{1} d f_{2} d f_{3} .
\end{aligned}
$$

where $\Lambda_{x x}^{\prime}\left(f_{1}, f_{2}, f_{3}\right)$ is defined in (55) in Appendix 7, and $\Lambda_{d x}^{\prime}\left(f_{1}, f_{2}, f_{3}\right)$ is

$$
\begin{aligned}
\Lambda_{d x}^{\prime}\left(f_{1}, f_{2}, f_{3}\right)= & \frac{\rho_{t}^{\frac{T_{s}}{2}}\left(1-\rho_{t}^{T_{s}}\right)\left(1+e^{j 2 \pi f_{3}}\right)}{1+\rho_{t}^{2 T_{s}}-2 \rho_{t}^{T_{s}} \cos \left(2 \pi f_{3}\right)} \\
& \cdot \sum_{i=-\infty}^{+\infty} \sum_{k=-\infty}^{+\infty} \rho_{s}^{\sqrt{\left[\left(i+\frac{1}{2}\right)^{2}+\left(k+\frac{1}{2}\right)^{2}\right] / \delta}} e^{-j 2 \pi\left(i f_{1}+k f_{2}\right)} .
\end{aligned}
$$




\section{Proof. The proof is in Appendix 8.}

Figure 9 compares the asymptotic MSE of the interpolation in a 2-D network with that in an 1-D network. In the simulation, the parameters are the same as those in Figure 7 except $\sigma_{w}^{2}=0$. In both 1-D and 2-D networks, it is observed that the interpolation MSE decreases monotonically with the temporal sampling rate. Again, the 1-D asymptotic MSE is smaller (better) than its 2D counterpart for all temporal correlation coefficients $\rho_{t}$. The performance difference between the 1-D and 2-D networks increases as $\rho_{t}$ increases.

If we just consider the spatial interpolation of the 2-D network, for the special case of uncorrelated data in the time domain, we can simplify the result as follows.

Corollary 8. If $\rho_{t}=0$, the asymptotic MSE of the estimated data during the spatial interpolations of the 2-D network with AWGN channels is

$$
\begin{aligned}
\varphi_{s}^{2}= & \int_{-\frac{1}{2}}^{\frac{1}{2}} \int_{-\frac{1}{2}}^{\frac{1}{2}}\left[\Lambda_{s s}\left(f_{1}, f_{2}\right)\right. \\
& \left.-\frac{\left|\Lambda_{d s}\left(f_{1}, f_{2}\right)\right|^{2}}{\Lambda_{s s}\left(f_{1}, f_{2}\right)+\sigma_{w}^{2}+\left(1+\sigma_{w}^{2}\right) \frac{\theta \delta}{\gamma_{0}}}\right] d f_{1} d f_{2},
\end{aligned}
$$

where $\Lambda_{s s}\left(f_{1}, f_{2}\right)$ is given in $(31)$, and $\Lambda_{d s}\left(f_{1}, f_{2}\right)$ is

$$
\Lambda_{d s}\left(f_{1}, f_{2}\right)=\sum_{i=-\infty}^{+\infty} \sum_{k=-\infty}^{+\infty} \rho_{s}^{\sqrt{\left[\left(i+\frac{1}{2}\right)^{2}+\left(k+\frac{1}{2}\right)^{2}\right] / \delta}} e^{-j 2 \pi\left(i f_{1}+k f_{2}\right)}
$$

Proof. The proof is in Appendix 9.

The result in Corollary 8 with $\sigma_{w}^{2}=0$ simplifies to ([6], Proposition 4), where only the spatial data correlation is considered.

\subsection{Optimum spatial-temporal sampling}

The asymptotically optimum spatial and temporal sampling rates in a 2-D network can be obtained by numerically solving $\left|\frac{\partial \varphi_{s t}^{2}}{\partial \delta}\right|=\epsilon_{s}$ and $\left|\frac{\partial \varphi_{s t}^{2}}{\partial \theta}\right|=\epsilon_{t}$, with $\epsilon_{s}$ and $\epsilon_{t}$ being very small numbers. Figure 10 shows the asymptotically optimum temporal sampling rate as a function of the temporal correlation coefficient in the 1-D and 2-D networks with AWGN channels. In the figure, $\rho_{s}=0.5, \sigma_{w}^{2}=0$, and $\epsilon=10^{-3}$ are used for both 1-D and 2-D networks. It is observed that the asymptotically optimum sampling rate for the 1-D and 2-D networks are almost identical, with the optimum sampling rate in the 1-D network slightly larger.

It should be noted that the analysis methods presented in this article can be extended to high dimensional networks by employing block multilevel Toeplitz matrix. In this article, the 1-D and 2-D networks are used as examples to investigate the interactions among the various network parameters and their impacts on the system performance. The results of high dimensional networks can be obtained in a similar manner.

\subsection{Randomly distributed networks}

So far all the studies are for networks with deterministic topologies. In this section, we will compare the

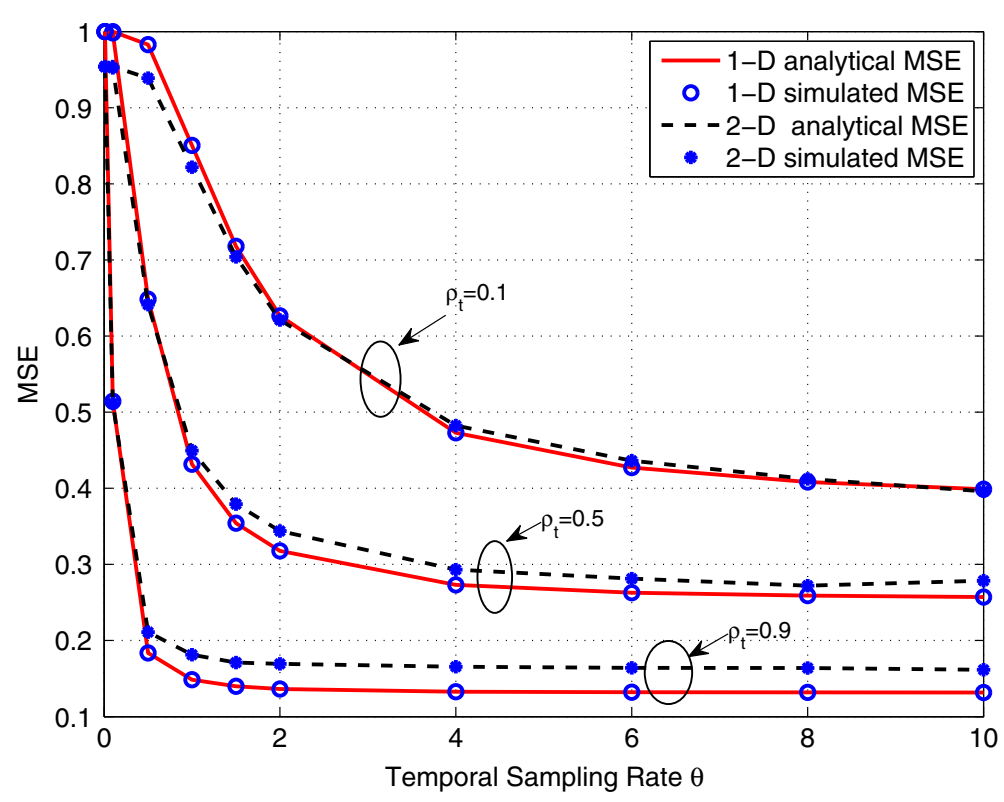

Figure 9 The asymptotic MSE of space-time interpolations in the 1-D and 2-D networks (AWGN, $\rho_{s}=0.5, \sigma_{w}^{2}=0, \gamma_{0}=0 \mathrm{~dB}$ ). 


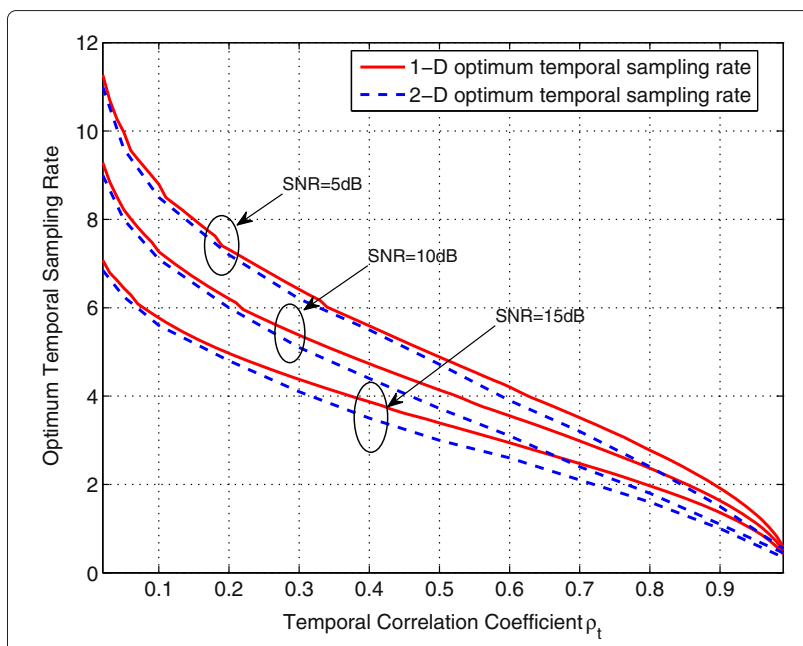

Figure 10 The asymptotically optimum sampling rate in the 1-D and 2-D networks (AWGN, $\rho_{s}=0.5, \sigma_{w}^{2}=0, \epsilon=10^{-3}$ ).

MSE performance between networks with deterministic topology and random topology, respectively. The random topology follows a Poisson point process, i.e., the number of nodes in a given area follows a Poisson distribution, and the coordinates of each node follows a uniform distribution in each dimension.

The MSE of the 1-D and 2-D networks with random topology can be evaluated numerically through a hybrid analytical-simulation method. The MSE conditioned on a particular deployment of the nodes can be calculated by using (12) for the 1-D network, or (29) for the 2-D network. The elements in the autocorrelation matrix, $\mathbf{R}_{x x}$ or $\boldsymbol{\Phi}_{x x}$, depends on the actual locations of the nodes. The unconditional MSE can then be calculated by averaging a large number of random deployments.

Figure 4.3 compares the performance of networks with random topology and deterministic topology, operating in AWGN channels. The parameters are $\gamma_{0}=10 \mathrm{~dB}$ and $\sigma_{w}^{2}=0$. For both 1 -D and 2-D networks, networks with deterministic topology consistently outperform their random topology counterparts. The difference between the two types of networks becomes smaller as $\rho_{s}$ and $\rho_{t}$ increase. The topology of practical networks is usually a combination of the grid-based deterministic topology and random topology. Therefore, the performance of practical networks will fall between the bounds delimited by the two types of networks.

\section{Conclusions}

In this article, the optimum sampling in the 1-D and 2-D WSNs with spatial-temporally correlated data was studied. The impacts of the spatial node density and the temporal sampling rate on the network performance were investigated through asymptotic analysis and numerical studies. Under the constraint of fixed power per unit area,

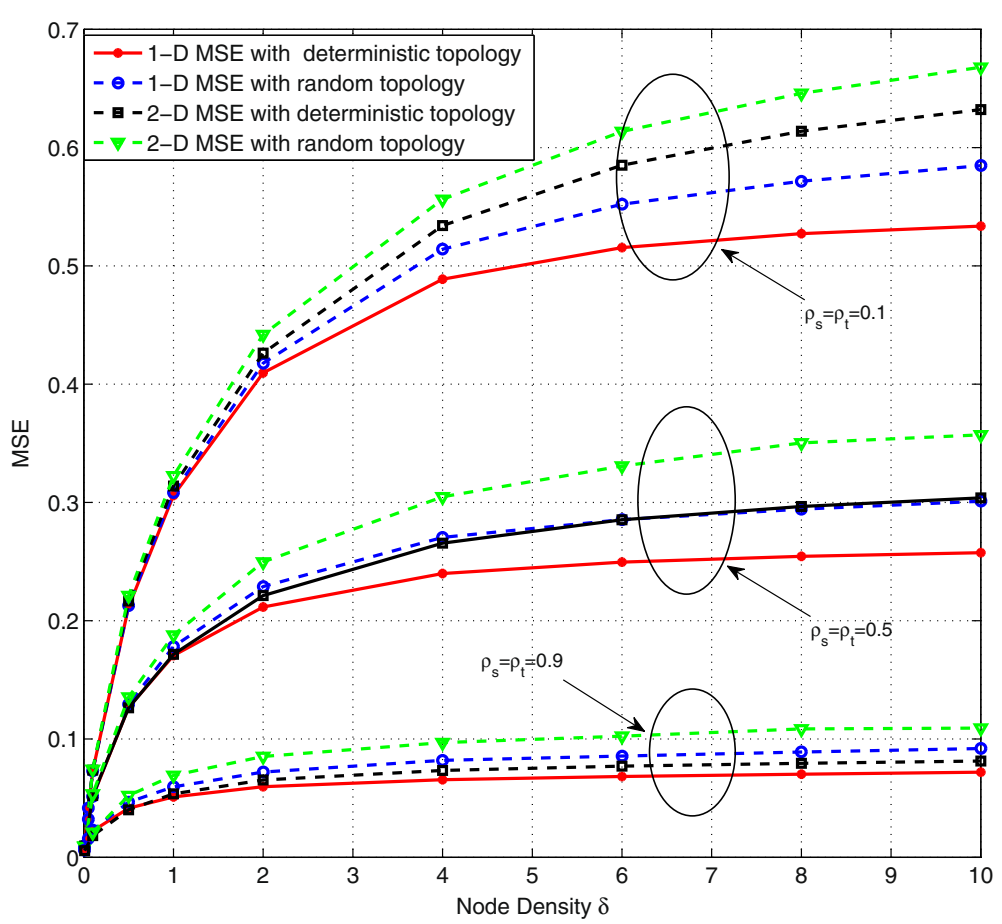

Figure 11 Comparison between networks with deterministic topology and random topology (AWGN, $\gamma_{0}=10 \mathrm{~dB}, \theta=10 \mathrm{~Hz}, \sigma_{w}^{2}=0$ ). 
the MSE performance of various networks were studied through a combination of analytical and simulation methods. The results quantitatively identified the interactions between the estimation fidelity and a large number of system parameters, such as node density, sampling rate, measurement noise, fading, and random topology, etc. It was observed that the network with a deterministic gridbased topology and operating in AWGN channels has the best performance, yet that with a random topology and operating in fading channels has the worst performance. Therefore, whenever possible, a grid-based deterministic topology is preferred over a random topology. The MSE performance of these two types of networks can serve as lower and upper bounds for practical networks, and their difference gradually diminish as the correlation coefficients increase.

\section{Appendix 1}

\section{Proof of Proposition 1}

Setting $\mathbf{H}=\mathbf{I}_{N}$ in (12) and performing the eigenvalue decomposition of $\mathbf{R}_{x x}$, we can rewrite the MSE as

$$
\sigma_{s t, N}^{2}=\frac{1}{N} \sum_{m=1}^{N_{s}-1} \sum_{k=1}^{N_{t}-1}\left(\frac{1}{\lambda_{m, k}}+\frac{1}{\sigma_{w}^{2}+\left(1+\sigma_{w}^{2}\right) \frac{\theta \delta}{\gamma_{0}}}\right)^{-1}
$$

where $\lambda_{m, k}$, for $m=0,1, \ldots, N_{s}-1$, and $k=0,1, \ldots, N_{t}-$ 1 , are the eigenvalues of $\mathbf{R}_{x x}$. When $N_{s} \rightarrow \infty$ and $N_{t} \rightarrow$ $\infty$, the 2-D discrete-time Fourier transform (DTFT) of the sequence, $\left\{\rho_{s}^{|m| d} \rho_{t}^{|k| T_{s}}\right\}_{m, k}$, which are elements of the TBT matrix $\mathbf{R}_{x x}$, can be calculated as $\Lambda_{x x}\left(f_{1}, f_{2}\right)=\Lambda\left(\rho_{s}^{d}, f_{1}\right) \times$ $\Lambda\left(\rho_{t}{ }^{T_{s}}, f_{2}\right)$, where

$$
\Lambda(a, f)=\sum_{m=-\infty}^{+\infty} a^{|m|} e^{-j 2 \pi m f}=\frac{1-a^{2}}{1+a^{2}-2 a \cos \left(2 \pi f_{1}\right)}
$$

Based on the extension of the Szego's theorem to TBT matrices ([18], Theorem 1), when $N_{s} \rightarrow \infty$ and $N_{t} \rightarrow \infty$, the asymptotic MSE is

$$
\begin{aligned}
\sigma_{s t}^{2} & =\lim _{N \rightarrow \infty} \sigma_{s t, N}^{2} \\
& =\int_{-\frac{1}{2}}^{\frac{1}{2}} \int_{-\frac{1}{2}}^{\frac{1}{2}}\left[\frac{1}{\Lambda_{x x}\left(f_{1}, f_{2}\right)}+\frac{1}{\sigma_{w}^{2}+\left(1+\sigma_{w}^{2}\right) \frac{\theta \delta}{\gamma_{0}}}\right]^{-1} d f_{1} d f_{2},
\end{aligned}
$$

Substituting the result of $\Lambda_{x x}\left(f_{1}, f_{2}\right)$ into (41), and applying ([19], Equation (2.553.3)), we can solve the inner integral as $\sigma_{s t}^{2}=\frac{1-\rho_{t}^{2 T_{s}}}{2 \rho_{t}^{T_{s}}} \int_{-\frac{1}{2}}^{\frac{1}{2}}\left[\cos ^{2}\left(2 \pi f_{2}\right)-a_{2} \cos \left(2 \pi f_{2}\right)+b_{2}\right]^{-\frac{1}{2}} f_{2}$,

where

$$
\begin{gathered}
a_{2}=\left[1+\rho_{t}^{2 T_{s}}+\frac{1}{\sigma_{w}^{2}+\left(1+\sigma_{w}^{2}\right) \frac{\theta \delta}{\gamma_{0}}} \cdot \frac{1+\rho_{s}^{2 d}}{1-\rho_{s}^{2 d}} \cdot\left(1-\rho_{t}^{2 T_{s}}\right)\right] \cdot \rho_{t}^{-T_{s}} \\
b_{2}=\left[\frac{2\left(1+\rho_{t}^{2 T_{s}}\right)^{2}}{\sigma_{w}^{2}+\left(1+\sigma_{w}^{2}\right) \frac{\theta \delta}{\gamma_{0}}} \cdot \frac{1+\rho_{s}^{2 d}}{1-\rho_{s}^{2 d}} \cdot\left(1-\rho_{t}^{4 T_{s}}\right)\right. \\
\left.+\left(\frac{1-\rho_{t}^{2 T_{s}}}{\sigma_{w}^{2}+\left(1+\sigma_{w}^{2}\right) \frac{\theta \delta}{\gamma_{0}}}\right)^{2}\right] \cdot\left(4 \rho_{t}^{-2 T_{s}}\right) .
\end{gathered}
$$

Based on ([19], Equation (2.580.2)) and ([19], Equation (3.152.2)), we can get the results in (13).

\section{Appendix 2}

Proof of Corollary 2

The MSE in (13) can be alternatively written as

$$
\sigma_{s t}^{2}=\frac{\sqrt{2}}{\pi} \cdot \int_{0}^{\frac{\pi}{2}} \frac{1}{\sqrt{\beta-\alpha \sin ^{2} x}} d x
$$

Since integration is a linear operator, we can directly find the limit of the integrand, and the result is

$$
\begin{aligned}
\lim _{\delta \rightarrow \infty}\left(\frac{1}{\sqrt{\beta-\alpha \sin ^{2} x}}\right) \\
=\sqrt{2 \log \left(\rho_{s}\right)}\left[\log \left(\rho_{s}\right)-\frac{2 \gamma_{0}}{\theta\left(1+\sigma_{w}^{2}\right)} \cdot \frac{1+\rho_{t}^{\frac{1}{\theta}}}{1-\rho_{t}^{\frac{1}{\theta}}}\right. \\
\left.-\frac{8 \gamma_{0}}{\theta\left(1+\sigma_{w}^{2}\right)} \cdot \frac{\rho_{t}^{\frac{1}{\theta}}}{1-\rho_{t}^{\frac{2}{\theta}}} \sin ^{2} x\right]^{-\frac{1}{2}} .
\end{aligned}
$$

Substituting (45) into (44) and simplifying lead to (16).

\section{Appendix 3}

\section{Proof of Corollary 4}

The limit in (17) can be rewritten as

$$
\begin{gathered}
\frac{2}{\pi}\left(1+\frac{4}{\varpi}\right)^{-\frac{1}{2}} \cdot K\left(\sqrt{\frac{4}{4+\varpi}}\right) \\
=\frac{2}{\pi} \int_{0}^{\frac{\pi}{2}} \frac{d w}{\sqrt{1+\frac{4}{\varpi}\left(1-\sin ^{2} w\right)}} .
\end{gathered}
$$


Since $\left(1-\sin ^{2} w\right)$ is a non-negative real number, the limit is an increasing function of $\varpi$, thus proportional to $\rho_{s}$ and $\rho_{t}$, but inverse proportional to the SNR $\gamma_{0}$.

\section{Appendix 4}

\section{Proof of Proposition 2}

The Toeplitz matrix, $\mathbf{R}_{s}^{\prime}$, is uniquely determined by the sequence $\left\{\rho_{s}^{\left|m+\frac{1}{2}\right| d}\right\}_{m=-\left(N_{s}-1\right)}^{\left(N_{s}-1\right)}$. Similarly, the Toeplitz matrix, $\mathbf{R}_{t}^{\prime}$, is uniquely determined by the sequence, $\left\{\rho_{t}^{\left|m+\frac{1}{2}\right| T_{s}}\right\}_{m=-\left(N_{t}-1\right)}^{\left(N_{t}-1\right)}$. Based on [21], when $N_{s} \rightarrow \infty$ and $N_{t} \rightarrow \infty$, the 2-D DTFT of the sequence, $\left\{\rho_{s}^{\left|m+\frac{1}{2}\right| d} \rho_{t}^{\left|k+\frac{1}{2}\right| T_{s}}\right\}_{m, k}$, which are elements of the TBT matrix $\mathbf{R}_{d x}$, can be calculated as $\Lambda_{d x}\left(f_{1}, f_{2}\right)=\Lambda^{\prime}\left(\rho_{s}{ }^{d}, f_{1}\right) \times$ $\Lambda^{\prime}\left(\rho_{t} T_{s}, f_{2}\right)$, where

$$
\Lambda^{\prime}(a, f)=a^{\frac{1}{2}} \frac{(1-a)\left(1+e^{j 2 \pi f}\right)}{1+a^{2}-2 a \cos (2 \pi f)}
$$

Based on ([18], Lemma 1$), \mathbf{R}_{d x}$ is asymptotically equivalent to a circulant-block-circulant (CBC) matrix, $\mathbf{C}_{d x}=$ $\mathbf{U}_{N}^{H} \mathbf{D}_{d x} \mathbf{U}_{N}$, where $\mathbf{U}_{N}^{H}$ is the unitary discrete Fourier transform (DFT) matrix and $\mathbf{D}_{d x}$ is a diagonal matrix with its $k$ th diagonal element being

$$
\left(\mathbf{D}_{d x}\right)_{k, k}=\Lambda^{\prime}\left(\rho_{s}^{d}, \frac{k-1}{N_{s}}\right) \cdot \Lambda^{\prime}\left(\rho_{t}^{T_{s}}, \frac{k-1}{N_{t}}\right) .
$$

Similarly, the TBT matrix, $\mathbf{R}_{x x}$, is asymptotically equivalent to a CBC matrix, $\mathbf{C}_{x x}=\mathbf{U}_{N}^{H} \mathbf{D}_{x x} \mathbf{U}_{N}$, where $\mathbf{D}_{x x}$ is a diagonal matrix with its $k$ th diagonal element being $\left(\mathbf{D}_{x x}\right)_{k, k}=\Lambda\left(\rho_{s}^{d}, \frac{k-1}{N_{s}}\right) \cdot \Lambda\left(\rho_{t}^{T_{s}}, \frac{k-1}{N_{t}}\right)$, with $\Lambda\left(\rho^{d}, f\right)$ defined in (40).

In addition, the $\mathrm{CBC}$ matrices, $\mathbf{C}_{x x}$ and $\mathbf{C}_{d x}$, share the same orthonormal eigenvectors [22]. Based on ([23], Theorem 2.1), the error correlation matrix, $\mathbf{R}_{e e}^{(d)}$, is asymptotically equivalent to a CBC matrix, $\mathbf{C}_{e e}^{(d)}=\mathbf{C}_{x x}-\mathbf{C}_{d x}\left(\mathbf{C}_{x x}+\left(\sigma_{w}^{2}+\frac{\theta \delta}{\gamma_{0}}\right) \mathbf{I}\right)^{-1} \mathbf{C}_{d x}^{H}=$ $\mathbf{U}_{N}^{H} \mathbf{D}_{e e}^{(d)} \mathbf{U}_{N}$, where the diagonal matrix $\mathbf{D}_{e e}^{(d)}=\mathbf{D}_{x x}-$ $\mathbf{D}_{d x}\left(\mathbf{D}_{x x}+\left(\sigma_{w}^{2}+\frac{\theta \delta}{\gamma_{0}}\right) \mathbf{I}_{N}\right)^{-1} \mathbf{D}_{d x}^{H}$.

Based on the extension of the Szego's theorem to TBT matrices ([18], Theorem 1), we have

$$
\begin{aligned}
\vartheta_{s t}^{2}= & \int_{-\frac{1}{2}}^{\frac{1}{2}} \int_{-\frac{1}{2}}^{\frac{1}{2}}\left[\Lambda_{x x}\left(f_{1}, f_{2}\right)\right. \\
& \left.-\frac{\left|\Lambda_{d x}\left(f_{1}, f_{2}\right)\right|^{2}}{\Lambda_{x x}\left(f_{1}, f_{2}\right)+\sigma_{w}^{2}+\left(1+\sigma_{w}^{2}\right) \frac{\theta \delta}{\gamma_{0}}}\right] d f_{1} d f_{2} .
\end{aligned}
$$

With ([19], Equation (2.559.2)), we can solve the inner integral, and the result is

$$
\begin{aligned}
\vartheta_{s t}^{2}= & \int_{-\frac{1}{2}}^{\frac{1}{2}}\left[\Lambda\left(\rho_{t}^{T_{s}}, f\right)-\frac{\left|\Lambda^{\prime}\left(\rho_{t}^{T_{s}}, f\right)\right|^{2}}{\Lambda\left(\rho_{t}^{T_{s}}, f\right)}+\frac{\left|\Lambda^{\prime}\left(\rho_{t}^{T_{s}}, f\right)\right|^{2}}{\Lambda\left(\rho_{t}^{T_{s}}, f\right)}\right. \\
& \times\left(\sigma_{w}^{2}+\left(1+\sigma_{w}^{2}\right) \frac{\theta \delta}{\gamma_{0}}+\frac{1-\rho_{s}^{\frac{1}{\delta}}}{1+\rho_{s}^{\frac{1}{\delta}}} \Lambda\left(\rho_{t}^{T_{s}}, f\right)\right)^{\frac{1}{2}} \\
& \left.\times\left(\sigma_{w}^{2}+\left(1+\sigma_{w}^{2}\right) \frac{\theta \delta}{\gamma_{0}}+\frac{1+\rho_{s}^{\frac{1}{\delta}}}{1-\rho_{s}^{\frac{1}{\delta}}} \Lambda\left(\rho_{t}^{T_{s}}, f\right)\right)^{-\frac{1}{2}}\right] d f
\end{aligned}
$$

From ([19], Equation (2.558.2)), we get

$$
\int_{-\frac{1}{2}}^{\frac{1}{2}}\left[\Lambda\left(\rho_{t}^{T_{s}}, f_{2}\right)-\frac{\left|\Lambda^{\prime}\left(\rho_{t}^{T_{s}}, f_{2}\right)\right|^{2}}{\Lambda\left(\rho_{t}^{T_{s}}, f_{2}\right)}\right] d f_{2}=\frac{1-\rho_{t}^{\frac{1}{\theta}}}{1+\rho_{t}^{\frac{1}{\theta}}}
$$

Substituting (51) into (50) and simplifying lead to (22).

\section{Appendix 5}

\section{Proof of Proposition 3}

The result in (24) can be proved by following a procedure that is similar to the proof of Proposition 2. Since the interpolation is performed in the space domain alone, we can replace $\Lambda^{\prime}\left(\rho_{t}^{T_{s}}, f_{2}\right)$ with $\Lambda\left(\rho_{t}^{T_{s}}, f_{2}\right)$ in (50), and the result is

$$
\begin{aligned}
\vartheta_{s}^{2}=\int_{-\frac{1}{2}}^{\frac{1}{2}} & {\left[\Lambda\left(\rho_{t}^{T_{s}}, f_{2}\right)\left(\sigma_{w}^{2}+\left(1+\sigma_{w}^{2}\right) \frac{\theta \delta}{\gamma_{0}}+\frac{1-\rho_{s}^{\frac{1}{\delta}}}{1+\rho_{s}^{\frac{1}{\delta}}} \Lambda\left(\rho_{t}^{T_{s}}, f_{2}\right)\right)^{\frac{1}{2}}\right.} \\
& \left.\times\left(\sigma_{w}^{2}+\left(1+\sigma_{w}^{2}\right) \frac{\theta \delta}{\gamma_{0}}+\frac{1+\rho_{s}^{\frac{1}{\delta}}}{1-\rho_{s}^{\frac{1}{\delta}}} \cdot \Lambda\left(\rho_{t}^{T_{s}}, f_{2}\right)\right)-\frac{1}{2}\right] d f_{2}
\end{aligned}
$$

The above integral can be solved by using ([19], Equation (3.147.2)), ([19], Equation (3.151.2)), and the definition of $\Lambda\left(\rho_{t}^{T_{s}}, f_{2}\right)$ in (40), and the result is (24).

\section{Appendix 6}

Proof of Corollary 6

Setting $\delta \rightarrow \infty$ in (52) leads to

$$
\lim _{\delta \rightarrow \infty} \vartheta_{s}^{2}=\int_{-\frac{1}{2}}^{\frac{1}{2}}\left[\Lambda\left(\rho_{t}^{T_{s}}, f_{2}\right) \cdot\left(1-\frac{\Lambda\left(\rho_{t}^{T_{s}}, f_{2}\right)}{\log \left(\rho_{s}\right)}\right)^{-\frac{1}{2}}\right] d f_{2}
$$


The above integral can be solved by using ([19], Equation (3.147.2)).

\section{Appendix 7}

\section{Proof of Proposition 4}

Setting $\mathbf{H}=\mathbf{I}_{N}$ in (29) and performing the eigenvalue decomposition of $\boldsymbol{\Phi}_{x x}$ in (28), we have

$$
\psi_{s t, N}^{2}=\frac{1}{N} \sum_{i=1}^{M_{s}} \sum_{k=1}^{M_{s}} \sum_{m=1}^{N_{t}}\left(\frac{1}{\lambda_{i k m}}+\frac{1}{\sigma_{w}^{2}+\left(1+\sigma_{w}^{2}\right) \frac{\theta \delta}{\gamma_{0}}}\right)^{-1}
$$

where $\lambda_{i k m}$, for $i, k=1, \ldots, M_{s}$, and $m=1, \ldots, N_{t}$, are eigenvalues of $\boldsymbol{\Phi}_{x x}$. When $N_{s} \rightarrow \infty$ and $N_{t} \rightarrow \infty$, the 3D DTFT of the sequence, $\left\{\rho_{s}^{\sqrt{\left(i^{2}+k^{2}\right) / \delta}} \rho_{t}^{|m| T_{s}}\right\}_{i k m}$, which are elements of the 3-level Toeplitz matrix $\boldsymbol{\Phi}_{x x}$, can be calculated as

$$
\Lambda_{x x}^{\prime}\left(f_{1}, f_{2}, f_{3}\right)=\Lambda_{s s}\left(f_{1}, f_{2}\right) \times \Lambda\left(\rho_{t}^{T_{s}}, f_{3}\right)
$$

The result in (30) follows immediately from (55) and ([20], Theorem 1), which is the extension of the Szego's theorem to multilevel Toeplitz matrices.

\section{Appendix 8}

\section{Proof of Proposition 5}

According to ([20], Lemma 2), the multilevel Toeplitz matrices, $\boldsymbol{\Phi}_{x x}$ and $\boldsymbol{\Phi}_{d x}$, are asymptotically equivalent to multilevel circulant matrices, $\mathbf{B}_{x x}$ and $\mathbf{B}_{d x}$, respectively, where the eigenvalues of $\mathbf{B}_{x x}$ and $\mathbf{B}_{d x}$ are samples of $\Lambda_{x x}^{\prime}\left(f_{1}, f_{2}, f_{3}\right)$ in $(55)$ and $\Lambda_{d x}^{\prime}\left(f_{1}, f_{2}, f_{3}\right)$ in (36), respectively. In addition, the multilevel circulant matrices, $\mathbf{B}_{x x}$ and $\mathbf{B}_{d x}$, share the same orthonormal eigenvectors [20]. Once the asymptotic equivalence is established, the rest of the proof follows the same procedure as described in Appendix 5 for the 1-D case.

\section{Appendix 9}

\section{Proof of Corollary 8}

When $N_{s} \rightarrow \infty$ and $N_{t} \rightarrow \infty$, while keeping $\delta$ and $\theta$ finite, the asymptotic MSE of spatial interpolations in a 2-D network is

$$
\begin{aligned}
& \int_{-\frac{1}{2}}^{\frac{1}{2}} \int_{-\frac{1}{2}}^{\frac{1}{2}} \int_{-\frac{1}{2}}^{\frac{1}{2}}\left[\Lambda_{s s}\left(f_{1}, f_{2}\right) \Lambda_{t}\left(f_{3}\right)\right. \\
& \left.\quad-\frac{\left|\Lambda_{d s}\left(f_{1}, f_{2}\right) \Lambda_{t}\left(f_{3}\right)\right|^{2}}{\Lambda_{s s}\left(f_{1}, f_{2}\right) \Lambda_{t}\left(f_{3}\right)+\sigma_{w}^{2}+\left(1+\sigma_{w}^{2}\right) \frac{\theta \delta}{\gamma_{0}}}\right] d f_{1} d f_{2} d f_{3} .
\end{aligned}
$$

where $\Lambda_{s s}\left(f_{1}, f_{2}\right)$ is defined in (31) and $\Lambda_{d s}\left(f_{1}, f_{2}\right)$ is computed as in (38). When $\rho_{t}=0$, we have $\Lambda_{t}\left(f_{3}\right)=1$. Substituting $\Lambda_{t}\left(f_{3}\right)=1$ into (56) directly leads to (37).

\section{Competing interests}

The authors declare that they have no competing interests.

\section{Acknowledgements}

The material in this article was presented in part at the IEEE Global

Telecommunication Conference (Globecom) 2011. This study was supported in part by the National Science Foundation under Grants ECCS-0917041 and ECCS-1202075, and the Arkansas NASA EPSCoR Research Infrastructure Development grant.

Received: 27 June 2012 Accepted: 20 November 2012 Published: 04 January 2013

\section{References}

1. J Wu, G Zhou, in Proc. IEEE Intern. Conf. Commun ICC'11. A new ultra-low power wireless sensor network with integrated energy harvesting, data sensing, and wireless communication, (Kyoto, Japan, June 2011), pp. 1-5

2. JF Chamberland, W Veeravalli, How dense should a sensor network be for detection with correlated observations. IEEE Trans. Inf. Theory. 52, 5099-5106 (2006)

3. SD Servetto, in Proc. 40th Annual Allerton Conf. Commu., Control, and Computing. On the feasibility of large scale wireless sensor networks, (Urbana, Illinois, October 2002)

4. Y Sung, HV Poor, H Yu, in Proc. IEEE Sensor Array Multichannel Signal Processing Workshop. Optimal node density for two-dimensional sensor arrays, (Darmstadt, Germany, July 2008), pp. 271-274

5. J Wu, N Sun, in Proc. IEEE Global Telecommun. Conf, GLOBECOM'10. Optimal sensor density in a distortion-tolerant linear wireless sensor network, (Miami, Florida, December 2010), pp. 1-5

6. J Wu, N Sun, Optimum sensor density in distortion tolerant wireless sensor networks. IEEE Trans. Wirel. Commun. 11, 2056-2064 (2012)

7. MC Vuran, OB Akan, IF Akyildiz, Spatio-temporal correlation: theory and applications for wireless sensor networks. Comput. Netw. J. (Elsevier). 45(3), 245-261 (2004)

8. R Cristescu, M Vetterli, On the optimal density for real-time data gathering of spatio-temporal processes in sensor networks. IEEE Trans. Sig. Proc. 51, 159-164 (2003)

9. X Zhang, H Wang, FN Abdesselam, AA Khokhar, Distortion analysis for real-Time data collection of spatially temporally correlated data fields in wireless sensor networks. IEEE Trans. Veh. Technol. 58, 1583-1594 (2009)

10. S Bandyopadhyay, Q Tian, EJ Coyle, Spatio-temporal sampling rates energy efficiency in wireless sensor networks. IEEE Trans. Network. 13, 1339-1352 (2005)

11. M Gastpar, M Vetterli, Power spatio-temporal bandwidth distortion in large sensor networks. IEEE J. Sel. Areas Commun. 23, 745-755 (2005)

12. N Sun, J Wu, in Proc. IEEE Global Telecommun. Conf. Globecom'11. Optimum sampling in a spatial-temporally correlated wireless sensor network, (Houston, Texas, December 2011), pp. 1-5

13. Q Zhao, L Tong, Opportunistic carrier sensing for energy-efficient information retrieval in sensor networks EURASIP. J. Wirel. Commun. Network. 2005, 231-241 (2005)

14. S Appadwedula, V Veeravalli, D Jones, Energy-efficient detection in sensor networks. IEEE J. Sel. Area Commun. 23, 693-702 (2005)

15. JF Chamberland, W Veeravalli, Asymptotic results for decentralized detection in power constrained wireless sensor networks. IEEE J. Sel. Areas Commun. 22, 1007-1015 (2004)

16. J Wu, in Proc. IEEE Mil. Commun. Conf. MILCOM'09. Ultra-low power compressive wireless sensing for distributed wireless networks, (Massachusetts, Boston, November 2009), pp. 1-7

17. SM Kay, Fundamentals of Statistical Signal Processing, vol. I, Estimation Theory. (Prentice-Hall, NJ, 1993)

18. PA Voois, A theorem on the asymtotic eigenvalue distribution of Toeplitz-block-Toeplitz matrix. IEEE Trans. Sig. Proc. 44, 1837-1841 (1996)

19. IS Gradshteyn, IM Ryzhik, Table of Integrals, Series, and Products, 6th edn. (Academic Press, Waltham, Massachusetts, 2000) 
20. M Oudin, JP Delmas, Asymptotic generalized eigenvalue distribution of block multilevel toeplitz matrices. IEEE Trans. Sig. Proc. 57, 382-387 (2009)

21. H Gazzah, PA Regalia, JP Delmas, Asymptotic eigenvalue distribution of block Toeplitz matrices and application to blind SIMO channel identification. IEEE Trans. Inf. Theory. 47, 1243-1251 (2001)

22. T Mazancourt, D Gerlic, The inverse of a block-circulant matrix. IEEE Trans. Antennas Propag. AP-31, 808-810 (1983)

23. RM Gray, Toeplitz and Circulant Matrices: A Review, NOW Publishers (2006). (available online at http://ee.stanford.edu/gray/)

doi:10.1186/1687-1499-2013-5

Cite this article as: Sun and Wu: Optimum sampling in spatial-temporally correlated wireless sensor networks. EURASIP Journal on Wireless Communications and Networking 2013 2013:5.

\section{Submit your manuscript to a SpringerOpen ${ }^{\mathcal{O}}$ journal and benefit from:}

- Convenient online submission

- Rigorous peer review

- Immediate publication on acceptance

- Open access: articles freely available online

- High visibility within the field

- Retaining the copyright to your article

Submit your next manuscript at $>$ springeropen.com 